Simplified models of mixed dark matter

This content has been downloaded from IOPscience. Please scroll down to see the full text. JCAP02(2014)011

(http://iopscience.iop.org/1475-7516/2014/02/011)

View the table of contents for this issue, or go to the journal homepage for more

Download details:

IP Address: 131.215.71.79

This content was downloaded on 17/04/2014 at 14:35

Please note that terms and conditions apply. 


\title{
Simplified models of mixed dark matter
}

\section{Clifford Cheung and David Sanford}

California Institute of Technology,

Pasadena, CA 91125, U.S.A.

E-mail: clifford.cheung@caltech.edu,dsanford@caltech.edu

Received December 22, 2013

Accepted January 13, 2014

Published February 6, 2014

\begin{abstract}
We explore simplified models of mixed dark matter (DM), defined here to be a stable relic composed of a singlet and an electroweak charged state. Our setup describes a broad spectrum of thermal DM candidates that can naturally accommodate the observed DM abundance but are subject to substantial constraints from current and upcoming direct detection experiments. We identify "blind spots" at which the DM-Higgs coupling is identically zero, thus nullifying direct detection constraints on spin independent scattering. Furthermore, we characterize the fine-tuning in mixing angles, i.e. well-tempering, required for thermal freeze-out to accommodate the observed abundance. Present and projected limits from LUX and XENON1T force many thermal relic models into blind spot tuning, well-tempering, or both. This simplified model framework generalizes bino-Higgsino DM in the MSSM, singlino-Higgsino DM in the NMSSM, and scalar DM candidates that appear in models of extended Higgs sectors.
\end{abstract}

Keywords: dark matter theory, dark matter detectors

ArXiv ePrint: 1311.5896 


\section{Contents}

1 Introduction $\quad 1$

2 Model definitions $\quad 3$

2.1 Singlet-doublet fermion 5

2.2 Singlet-doublet scalar $\quad 7$

2.3 Singlet-triplet scalar 11

3 Model A: singlet-doublet fermion DM 13

$3.1 \quad$ Exclusion plots (general) 13

$\begin{array}{lll}3.2 & \text { Exclusion plots (thermal relic) } & 17\end{array}$

3.3 Exclusion plots (thermal relic and marginal exclusion) 21

4 Model B: singlet-doublet scalar DM 24

4.1 Exclusion plots (general) 25

4.2 Exclusion plots (thermal relic) 26

5 Model C: singlet-triplet scalar DM 29

5.1 Exclusion plots (general) 30

5.2 Exclusion plots (thermal relic) 31

6 Conclusions and future directions $\quad 34$

\section{Introduction}

The gravitational evidence for dark matter (DM) is very strong, but its precise particle properties remain elusive. Long ago, laboratory experiments excluded the simplest models of weakly interacting massive particle (WIMP) DM which predicted DM-nucleon scattering via spin independent $Z$ boson exchange. Today, direct detection experiments, particularly XENON100 [1, 2] and LUX [3], have become sensitive to the large class of theories that predict spin independent (SI) DM-nucleon scattering mediated by the Higgs boson. Future experiments such as XENON1T [4] and LZ [5] will have improved sensitivities and their results will have even stronger implications. In light of the discovery of the Higgs boson at the LHC [6, 7], present and future limits on Higgs-mediated scattering can be recast in terms of the effective parameter space defined by the DM mass, $M_{\chi}$, and its coupling to the Higgs boson, $c_{h \chi \chi}$.

What are the natural values for $M_{\chi}$ and $c_{h \chi \chi}$ ? In the absence of additional theory input, these parameters are arbitrary - there is simply no reason why DM should be accessible through direct detection. For example, DM could be completely inert and thus impervious to non-gravitational probes. While many theories offer a DM candidate as part of a new physics framework, the only general impetus for couplings between DM and the Standard Model (SM) is cosmological in nature: if DM is a thermal relic, then it is reasonable for it be thermalized with the SM in the early universe. In minimal extensions of the SM, DM couples via electroweak gauge interactions and/or via the Higgs portal. Going beyond this setup requires more elaborate models that entail richer structures like dark force carriers [8-15] or 
other mediators $[16,17]$. Thus, an important question for present and upcoming experiments is the status of thermal relic DM, broadly defined.

The literature provides a litany of well-motivated theories of DM, both within and outside of broader new physics frameworks, though by far the most popular is neutralino DM in supersymmetry (SUSY). While supersymmetric theories are a useful benchmark for models, analyses of SUSY DM are often colored by theory biases and disparate connections to unrelated experimental data. For instance, under specific model assumptions, issues of naturalness are still taken as a hard constraint on the parameter space of SUSY DM models. Another example is the discrepancy in $g-2$ of the muon [18-20], which prefers certain signs of the $\mu$ parameter, influencing the perceived viability of SUSY DM. However, given the current sensitivity of experiments, overarching theory assumptions like SUSY are not required to pare down the parameter space - experiments will do so.

Instead it can be fruitful to take the approach of simplified models: effective theories that describe a broad class of theories but are tailored to extract maximal information from experimental results. Simplified models of DM have appeared in the literature in a number of guises. In the case of minimal DM [21, 22], pure gauge representations were considered. Others have studied simplified models of a singlet and colored particle, a.k.a. the effective "bino-squark" system [23-25]. For thermal relics, it was found that many of these models are bowing under the weight of present experimental constraints from direct detection and the LHC. Recently, there has also been growing interest in effective operator descriptions of DM [26-31]. Modulo the well-known limits of their validity [27, 29, 32-34], these effective theories have been used to determine quite general bounds on DM from colliders.

In the present work, we consider simplified models of mixed DM, defined here as renormalizable theories of fermion or scalar DM comprised of a singlet and an additional electroweak charged state. Generically, the singlet and charged states will mix after electroweak symmetry breaking. As a consequence, the DM possesses annihilation channels inherited from its electroweak charged component, and thermal relic DM can be achieved with an appropriate degree of mixing. In a sense, this simplified model is a generalization of the "welltempered" neutralino [35-37] found most commonly in focus point SUSY scenarios [38-41] to a more diverse set of DM charges and spins. The model still only possesses a singlet dark matter particle, in contrast to "multi-component" DM with similar field content $[42,43]$. By enumeration, there exist three renormalizable, gauge invariant simplified models of mixed dark matter: fermion singlet-doublet (Model $A$ ), scalar singlet-doublet (Model B), and scalar singlet-triplet (Model C). More complicated models necessarily include additional degrees of freedom or higher dimension operators to induce mixing. We evaluate the viability of models based upon current limits at LUX [3] and the expected reach of XENON1T [4]. Our main conclusions are:

- In light of current LUX limits and the projected reach of XENON1T, we have determined the viable parameter space of thermal relic DM in figure 9 (singlet-doublet fermion), figure 15 (singlet-doublet scalar), and figure 19 (singlet-triplet scalar). We have cast our results in terms of the parameter space of physical quantities: the DM mass, $M_{\chi}$, and the DM-Higgs coupling, $c_{h \chi \chi}$.

- Model A: singlet-doublet fermion. LUX stringently constrains this model except in regions with relative signs among the DM-Higgs Yukawa couplings. Given the overall Yukawa coupling strength $y$ defined in section 2.1, XENON1T will eliminate all of the viable parameter space with $y \gtrsim 0.1$ except near blind spots with tuning more severe 
than at least $\lesssim 10 \%$. XENON1T will allow for regions with $y \lesssim 0.1$, but they require $\lesssim 10 \%$ tuning in order to accommodate the observed relic density.

- Model B: singlet-doublet scalar. LUX places modest limits on this model but leaves much of the parameter space still open. Given the overall DM-Higgs quartic coupling strength $\lambda$ defined in section 2.2, XENON1T will eliminate essentially all of the parameter space for $\lambda \leq 0$. For $\lambda>0$, blind spots appear but a typical tuning of $\lesssim 10 \%$ is still required to escape the projected reach of XENON1T. Within the limits of our parameter scan, a similar tuning is required to obtain the observed relic abundance.

- Model C: Singlet-triplet scalar. LUX places relatively weak limits on the thermal relic parameter space because triplets annihilate very efficiently in the early universe. Given the overall DM-Higgs quartic coupling strength $\lambda$ defined in section 2.3, XENON1T will strongly constrain models with $\lambda \leq 0$, requiring a tuning of $\lesssim 1 \%$ to match the observed DM abundance except for nearly pure triplet DM. For $\lambda>0$, the coupling is suppressed and there are regions with all fine-tunings alleviated to $\gtrsim 10 \%$.

In section 2 we give a precise definition of Models $A, B$, and $C$, and discuss general aspects of the thermal relic abundance and the DM-nucleon scattering cross-section. For the latter, we derive analytic formulas indicating when the DM-Higgs coupling identically vanishes. In section 3 , section 4 , and section 5 , we examine the parameter spaces of Models $A, B$, and $C$, both in generality and for the case of thermal relic DM which saturates the observed abundance. Current bounds from LUX and projected reach of XENON1T bounds are shown throughout. Finally, in section 6 we present a discussion of our results and concluding thoughts.

\section{Model definitions}

In this section we explicitly define our simplified models. Throughout, we focus on the case of a singlet mixed with a non-singlet, which is a natural generalization of many models of theoretical interest. Of course, mixing among non-singlet states is also viable, but in this case the preferred mass range for DM is typically in the multi-TeV range, with a lower bound of several hundred $\mathrm{GeV}$.

Furthermore, we restrict our discussion to models with renormalizable interactions. While non-renormalizable interactions are of course allowed, they are competitive with renormalizable operators only when the cutoff is so low that the effective theory is invalid. Indeed, mixing induced by higher dimension operators is highly suppressed by the electroweak symmetry breaking scale divided by the cutoff scale. Even in scenarios where such a theory is valid and produces the appropriate relic density, a large degree of well-tempering is required for even marginal mixing, disfavoring it for this study. Restricting to renormalizable models limits us to three simplified models of mixed DM:

- Model A: Majorana fermion DM composed of a Majorana fermion singlet and Dirac fermion doublet with hypercharge $Y=1 / 2$.

- Model B: real scalar DM composed of a real scalar singlet and complex scalar doublet with hypercharge $Y=1 / 2$.

- Model C: real scalar DM composed of a real scalar singlet and real scalar triplet with hypercharge $Y=0$. 
In principle, one can consider singlets which are Dirac fermions or complex scalars, but these theories have more degrees of freedom and the analysis does not qualitatively change.

Throughout, we take $\Omega_{\chi}$ to be the relic abundance for the DM predicted by a thermal history. In all cases, the relic abundance and direct detection cross-section are calculated with MICROMEGAs 2.4.5 [44-46] using model files generated using FEYNRULES 1.6.11 [47]. We are predominantly interested in the parameter space that saturates the DM abundance observed by Planck [48],

$$
\Omega_{\mathrm{DM}} h^{2} \simeq 0.1199 \pm 0.0027 .
$$

Obviously, the DM relic abundance will drastically vary if the cosmological history is nonthermal, and in such cases there is no requirement that DM couples to the SM at all. Our initial analysis for each model will highlight the location of the $\Omega_{\chi}=\Omega_{\mathrm{DM}}$ line in parameter space, along with regions of $\Omega_{\chi}<\Omega_{\mathrm{DM}}$ and $\Omega_{\chi}>\Omega_{\mathrm{DM}}$. Our more detailed analysis will be restricted to the $\Omega_{\chi}=\Omega_{\mathrm{DM}}$ region, examining the behavior of other observables within the thermal relic context.

In all of our models, the DM particle is either a real scalar or Majorana fermion. Consequently, SI scattering through the $Z$ boson is inelastic and can be ignored. On the other hand, SI scattering through the Higgs boson is mediated via mixing between the singlet and and non-singlet components, though it is suppressed at direct detection "blind spots": regions of parameter space at which the coupling of DM to the Higgs boson vanishes identically. As noted in [49], the existence of blind spots depend sensitively on relative signs among the DM parameters. In the blind spot parameter space, the SI scattering DM-nucleon cross-section is zero at tree-level. Radiative corrections are typically sub-dominant in the parameter space except very close to the blind spot cancellation points. However, a proper evaluation of these higher order effects may become important for the status of DM if direct detection experiments do not observe SI scattering [50,51]. We also neglect radiative corrections to the masses, which are important for the phenomenology of minimal DM [21] but are subdominant when large mixing effects are introduced.

In the spirit of low energy Higgs theorems, we can straightforwardly compute the coupling of DM to the Higgs via a Taylor expansion of the DM mass term with respect to the Higgs vacuum expectation value (VEV), $v$. For Majorana fermion DM, we obtain

$$
\begin{aligned}
-\mathcal{L} & =\frac{1}{2} M_{\chi}(v+h) \chi \chi \\
& =\frac{1}{2} M_{\chi}(v) \chi \chi+\frac{1}{2} \frac{\partial M_{\chi}(v)}{\partial v} h \chi \chi+\mathcal{O}\left(h^{2}\right),
\end{aligned}
$$

where $v=246 \mathrm{GeV}$. Eq. (2.3) implies a dimensionless DM-Higgs boson coupling given by $c_{h \chi \chi}=\partial M_{\chi}(v) / \partial v$. For real scalar DM the same formula applies except with the replacement $M_{\chi}(v) \rightarrow M_{\chi}^{2}(v)$. In this case we define a dimensionful coupling $a_{h \chi \chi}=\partial\left[M_{\chi}^{2}(v)\right] / \partial v$ which is proportional to the DM mass, though for ease of discussion we will sometimes use the effective dimensionless coupling, $c_{h \chi \chi}=a_{h \chi \chi} / M_{\chi}$ instead. As discussed in ref. [49], the blind spot is defined by $c_{h \chi \chi}=0$, computed by taking the $\partial / \partial v$ derivative of the characteristic eigenvalue equation for the DM mass.

The DM-Higgs coupling maps straightforwardly onto limits from direct detection. The spin independent DM-nucleon cross-section is mediated by Higgs exchange and scales as

$$
\sigma^{\mathrm{SI}} \propto \frac{\mu^{2}}{m_{h}^{4}} \times c_{h \chi \chi}^{2},
$$


where we use $m_{h}=125.6 \mathrm{GeV}$ throughout [6, 7]. Strictly speaking, both $\mu$ and $c_{h \chi \chi}$ vary with $M_{\chi}$. In our region of interest, though, we require $M_{\chi} \gtrsim 100 \mathrm{GeV}$ to avoid LEP constraints on additional charged states which accompany the DM particle. Thus, $\mu$ is approximately equal to the nucleon mass. Meanwhile, we can compare the $\sigma^{\text {SI }}$ computed from theory with the limits from LUX and XENON1T. These limits have complicated mass dependence at low mass due to reduced efficiency in observing low energy events. However, for $M_{\chi} \gtrsim 100 \mathrm{GeV}$, the cross-section bounds $\sigma_{\mathrm{LUX}}^{\mathrm{SI}}, \sigma_{\mathrm{X} 1 \mathrm{~T}}^{\mathrm{SI}}$ rise linearly with $M_{\chi}$ because the event rates are proportional to the DM density, which falls with $1 / M_{\chi}$. Throughout, we use the lattice values for the quark content of the nucleon from [52].

We will be interested in models which evade present and projected limits from direct detection while accommodating a thermal relic abundance consistent with observation. However, these theories may require tuning for either or both of these aspects. For direct detection, the DM-Higgs coupling is of the schematic form $c_{h \chi \chi}=a+b$, where $a$ and $b$ depend on different sets of model parameters. We can characterize the degree of tuning required for a blind spot cancellation by

$$
\xi_{\mathrm{BS}}=\frac{|a+b|}{|a|+|b|}
$$

Blind spot tuning grows more severe as $\xi_{\mathrm{BS}} \rightarrow 0$. This effectively captures the tuning inherent in $c_{h \chi \chi} \rightarrow 0$ when individual Higgs couplings remain non-zero. If $a$ and $b$ have the same sign, no cancellation in $c_{h \chi \chi}$ is possible, and $\xi_{\mathrm{BS}}=1$.

Meanwhile, achieving the correct thermal relic abundance may require fine-tuning of mixing angles, or "well-tempering", since the DM must be the appropriate admixture of singlet and non-singlet state. Heuristically, well-tempering is correlated with the existence of small mass splittings in the DM multiplet relative to the dimensionful input parameters. Concretely, if the mass squared matrix is an $N \times N$ matrix $\mathbf{M}^{2}$, then the severity of welltempering is linked to the relative size of the traceless component of $\mathbf{M}^{2}$ (the mass splittings) relative to the trace of $\mathbf{M}^{2}$ (the overall mass scale). Hence, we define a parameter that describes the well-tempering in the mixing angle,

$$
\xi_{\mathrm{WT}}=\left(\frac{N \operatorname{Tr}\left[\mathbf{M}^{4}\right]}{\operatorname{Tr}\left[\mathbf{M}^{2}\right]^{2}}-1\right)^{\frac{1}{2}}
$$

which is related to the variance of the $\mathbf{M}^{2}$ matrix. Indeed, $\xi_{\mathrm{WT}}$ is precisely the fractional standard deviation of the eigenvalues of $\mathbf{M}^{2}$. In the limit that the entire DM multiplet is exactly degenerate, the mixing angle is very fine-tuned, $\mathbf{M}^{2} \propto \mathbb{1}$ and $\xi_{\mathrm{WT}} \rightarrow 0$.

\subsection{Singlet-doublet fermion}

In this section we define a simplified model for fermionic mixed DM comprised of a Majorana singlet and Dirac doublet of $\mathrm{SU}(2)_{W} \times \mathrm{U}(1)_{Y}$. Here we have introduced a Dirac doublet in order to cancel anomalies and allow for a bare mass term. A priori, the Dirac doublet can have arbitrary hypercharge, but to induce mixing via renormalizable interactions, we demand that $Y= \pm 1 / 2$ so that either the left-handed or right-handed doublet component can couple to the Higgs and the singlet. The field content of this simplified model is 


\begin{tabular}{|c|c|c|}
\hline \multicolumn{3}{|c|}{ Model $A$} \\
\hline Field & Charges & Spin \\
\hline$S$ & $(\mathbf{1}, 0)$ & $1 / 2$ \\
\hline$D_{1}$ & $(\mathbf{2},-1 / 2)$ & $1 / 2$ \\
\hline$D_{2}$ & $(\mathbf{2}, 1 / 2)$ & $1 / 2$ \\
\hline
\end{tabular}

and the general renormalizable Lagrangian is

$$
-\mathcal{L}_{\text {Model } A}=\frac{1}{2} M_{S} S^{2}+M_{D} D_{1} D_{2}+y_{D_{1}} S H D_{1}+y_{D_{2}} S H^{\dagger} D_{2}+H . c .,
$$

where we have dropped the kinetic terms for simplicity. In our notation, $D_{1}$ and $D_{2}$ have the same quantum numbers as $\tilde{H}_{d}$ and $\tilde{H}_{u}$ of the MSSM, respectively. We will sometimes parametrize the Yukawa couplings in polar coordinates,

$$
\begin{aligned}
& y_{D_{1}}=y \cos \theta \\
& y_{D_{2}}=y \sin \theta .
\end{aligned}
$$

Throughout, we work in a convention where $M_{S}$ and $M_{D}$ are positive but $y_{D_{1}}$ and $y_{D_{2}}$ have indefinite sign. Note, however, that using a parity transformation $S \rightarrow-S$, we can simultaneously flip the signs of $y_{D_{1}}$ and $y_{D_{2}}$, so only their relative sign is physical. Likewise, a parity transformation $D_{1} \rightarrow-D_{1}$ or $D_{2} \rightarrow-D_{2}$ flips the signs of $M_{D}$ and either $y_{D_{1}}$ or $y_{D_{2}}$, respectively, so only a singlet sign among the three parameters is physical. After electroweak symmetry breaking, $S$ mixes with $D_{1}$ and $D_{2}$, and this mixing simultaneously controls the thermal relic density of the DM as well as its coupling to the Higgs boson. The lightest neutral state, $\chi$, is stable DM, and is defined as a linear combination of the interaction eigenstates, $\chi=N_{S} S+N_{D_{1}} D_{1}+N_{D_{2}} D_{2}$, where $N_{S}^{2}+N_{D_{1}}^{2}+N_{D_{2}}^{2}=1$. There is also an electrically charged state in the spectrum which we denote by $\chi^{ \pm}$. Two heavier neutral states are also present, but they have little effect except when coannihilation controls the thermal relic density.

Eq. (2.7) parametrizes a broad class of models which have been discussed in the literature. Most prominently, it describes bino-Higgsino DM in the MSSM, with $y=g^{\prime} / \sqrt{2}$ and $\theta=\beta$. It also describes singlino-Higgsino mixing in the NMSSM, where mixing is controlled by the superpotential term $W=\lambda S H_{u} H_{d}$, so $y=\lambda$ and $\theta$ and $\beta$ are offset by $\pi / 2$. Singlet-doublet Majorana DM has also been discussed in great detail in ref. [53], and a similar scenario with a dirac singlet was considered in ref. [54].

Next, we present an analytic derivation of the relevant properties of $\chi$. In the basis $\left(S, D_{1}, D_{2}\right)$, the neutral mass matrix is

$$
\mathbf{M}=\left(\begin{array}{ccc}
M_{S} & \frac{1}{\sqrt{2}} y_{D_{1}} v & \frac{1}{\sqrt{2}} y_{D_{2}} v \\
\frac{1}{\sqrt{2}} y_{D_{1}} v & 0 & M_{D} \\
\frac{1}{\sqrt{2}} y_{D_{2}} v & M_{D} & 0
\end{array}\right) .
$$

The characteristic equation of the mass matrix is

$$
\begin{aligned}
0 & =\left(M_{\chi}^{2}-M_{D}^{2}\right)\left(M_{S}-M_{\chi}\right)+M_{D} y_{D_{1}} y_{D_{2}} v^{2}+\frac{1}{2} M_{\chi}\left(y_{D_{1}}^{2}+y_{D_{2}}^{2}\right) v^{2} \\
& =\left(M_{\chi}^{2}-M_{D}^{2}\right)\left(M_{S}-M_{\chi}\right)+\frac{1}{2} y^{2} v^{2}\left(M_{\chi}+M_{D} \sin 2 \theta\right)
\end{aligned}
$$


where we are interested in the smallest eigenvalue, $M_{\chi}$. Since $y$ labels the overall magnitude of the Yukawa couplings, its sign is unphysical and the characteristic equation depends only on $y^{2}$. On the other hand, the sign of $y_{D_{1}} / y_{D_{2}}$ is physical, and is represented by the sign of $\tan \theta$ (or equivalently $\sin 2 \theta$ ). The DM-Higgs coupling, $c_{h \chi \chi}=\partial M_{\chi}(v) / \partial v$, can be computed exactly by differentiating the characteristic equation in eq. (2.11) with respect to $\partial / \partial v$ and solving, yielding

$$
c_{h \chi \chi}=-\frac{y^{2} v\left(M_{\chi}+M_{D} \sin 2 \theta\right)}{M_{D}^{2}+2 M_{S} M_{\chi}-3 M_{\chi}^{2}+y^{2} v^{2} / 2} .
$$

We define "blind spot" for spin independent direct detection by all parameter points which satisfy $c_{h \chi \chi}=0$, so

$$
M_{\chi}+M_{D} \sin 2 \theta=0,
$$

as discussed in [49]. Because $M_{S}$ and $M_{D}$ are positive definite, a blind spot can only occur when $\sin 2 \theta<0$.

In general, we will be interested in the amount of tuning required of thermal relic DM permitted by current and future direct detection sensitivity. Using the general expressions for the tuning measures in eq. (2.5) and eq. (2.6), we define

$$
\begin{aligned}
\xi_{\mathrm{WT}} & =\frac{\sqrt{2\left(M_{D}^{2}-M_{S}^{2}\right)^{2}+\frac{1}{2} y^{4} v^{4}+2 y^{2} v^{2}\left[M_{D}^{2}+2 M_{S}^{2}+3 M_{D} M_{S} \sin 2 \theta\right]}}{2 M_{D}^{2}+M_{S}^{2}+y^{2} v^{2}} \\
\xi_{\mathrm{BS}} & =\left|\frac{M_{\chi}+M_{D} \sin 2 \theta}{M_{\chi}+M_{D}|\sin 2 \theta|}\right|
\end{aligned}
$$

corresponding to the amount of tuning required for a properly well-tempered thermal relic, and to the amount of tuning required to reside sufficiently close to a blind spot cancellation, respectively.

Finally, let us consider the DM mass and mixing angles in the parameter space of singletdoublet DM. Figures 1 and 2 show contours of $M_{\chi}$ and DM-singlet mixing angle squared, $N_{S}^{2}$, in the $\left(M_{D}, M_{S}\right)$ plane for $\tan \theta= \pm 2, \pm 10$ for $y=0.3$ and $y=1.5$, respectively. For $y=0.3$, $M_{\chi}$ is equal to the lower of $M_{S}$ or $M_{D}$ in most of the parameter space, only deviating near $M_{S} \approx M_{D}$. Significant mixing occurs when $M_{S} \approx M_{D}$, and away from this region the DM quickly approaches a pure singlet or pure doublet. For $y=1.5, M_{\chi}$ is significantly offset from both $M_{S}$ and $M_{D}$ throughout the plotted range for $\tan \theta>0$, with a larger offset around $M_{S} \approx M_{D}$. A sizable region even exists with $M_{\chi}<100 \mathrm{GeV}$ for low values of $M_{S}$ and/or $M_{D}$. For $\tan \theta<0$, however, the offset is more modest. As indicated, the degree of mixing typically much greater for $y=1.5$ than for $y=0.3$.

\section{$2.2 \quad$ Singlet-doublet scalar}

Next, we define another simplified DM model with singlet-doublet mixing, only with scalars rather than fermions. As in the case of fermionic DM, singlet-doublet mixing for scalars requires that the doublet have hypercharge $Y=1 / 2$. However, in this case a second doublet is not required to either accommodate a bare doublet mass or cancel anomalies. The field content is 


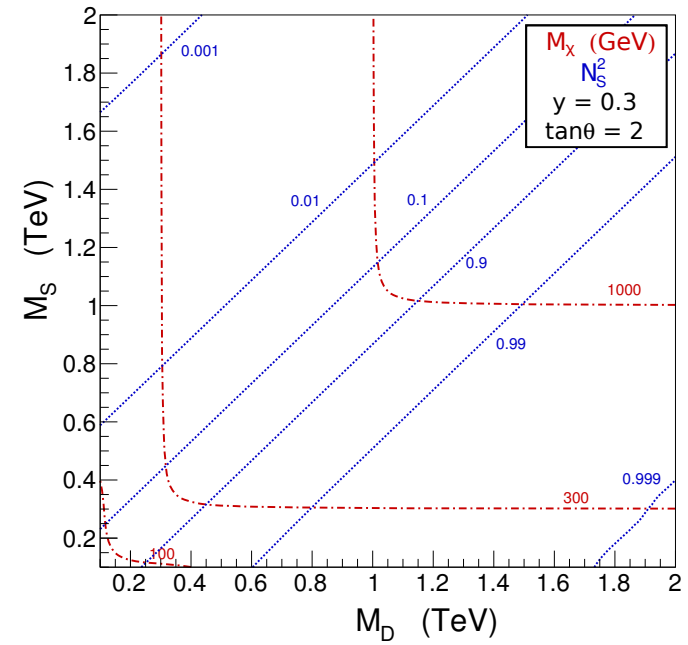

(a)

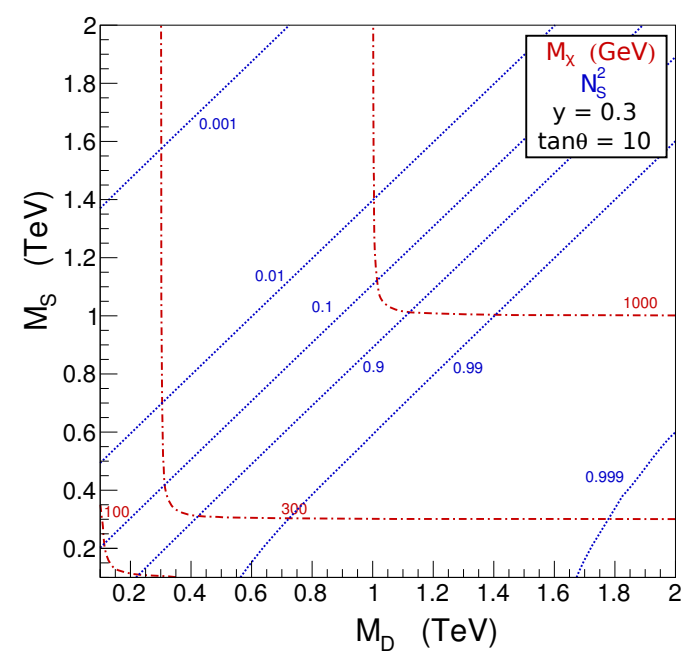

(c)

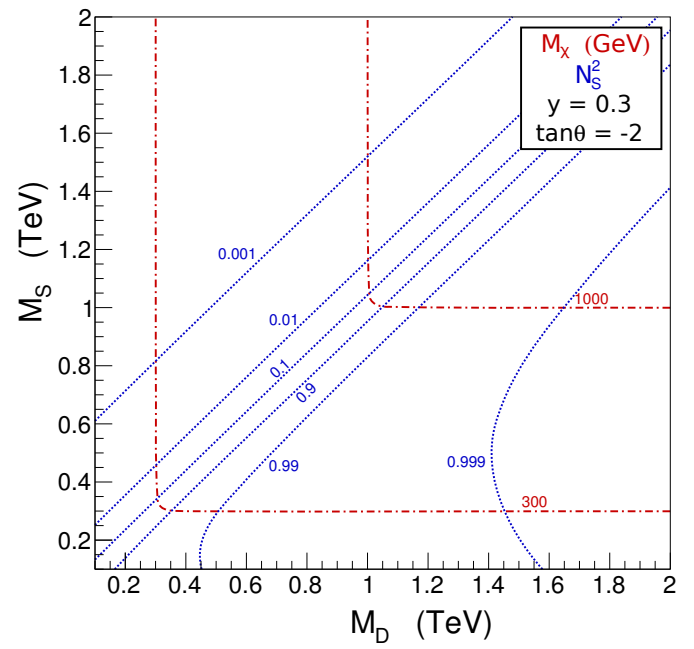

(b)

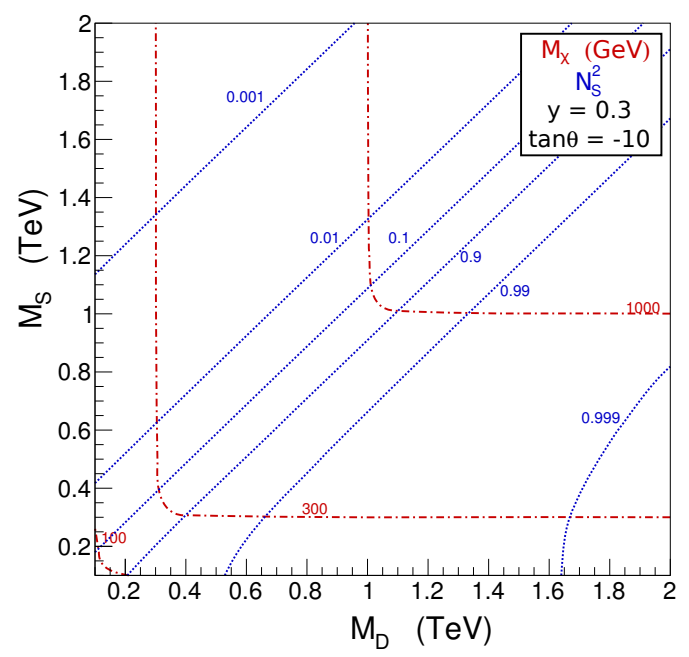

(d)

Figure 1. Mass and mixing angle contours in singlet-doublet fermion DM for $y=0.3$ and $\tan \theta=$ $\pm 2, \pm 10$. Shown are contours of the DM mass $M_{\chi}$ in $\mathrm{GeV}$ (red dot-dashed) and the DM-singlet mixing angle squared $N_{S}^{2}$ (blue dotted).

\begin{tabular}{|c|c|c|}
\hline \multicolumn{3}{|c|}{ Model B } \\
\hline Field & Charges & Spin \\
\hline$S$ & $(\mathbf{1}, 0)$ & 0 \\
\hline$D$ & $(\mathbf{2}, 1 / 2)$ & 0 \\
\hline
\end{tabular}

with a corresponding Lagrangian

$$
\begin{aligned}
-\mathcal{L}_{\text {Model B }}= & \frac{1}{2} M_{S}^{2} S^{2}+M_{D}^{2}|D|^{2}+\frac{1}{2} \lambda_{S} S^{2}|H|^{2}+\lambda_{D}|D|^{2}|H|^{2}+\lambda_{D}^{\prime}\left|H D^{\dagger}\right|^{2} \\
& +\frac{1}{2} \lambda_{D}^{\prime \prime}\left[\left(H D^{\dagger}\right)^{2}+\text { h.c. }\right]+A\left[S H D^{\dagger}+\text { h.c. }\right] .
\end{aligned}
$$




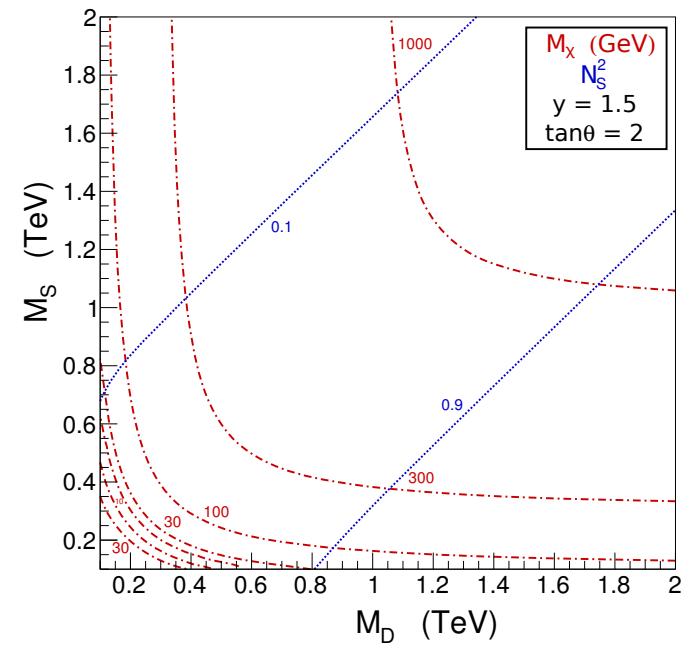

(a)

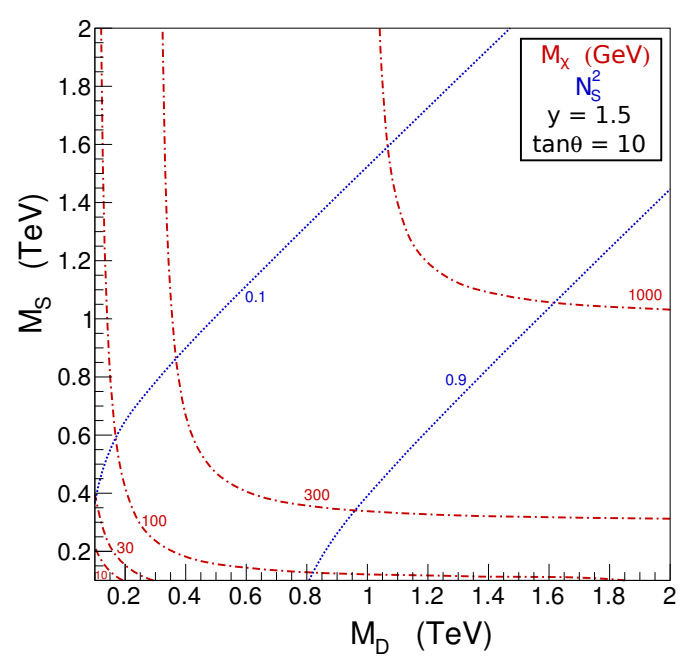

(c)

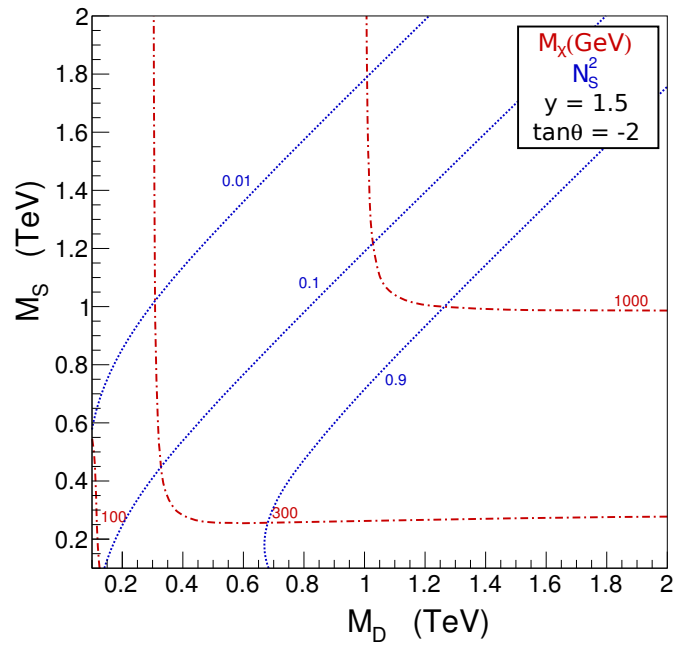

(b)

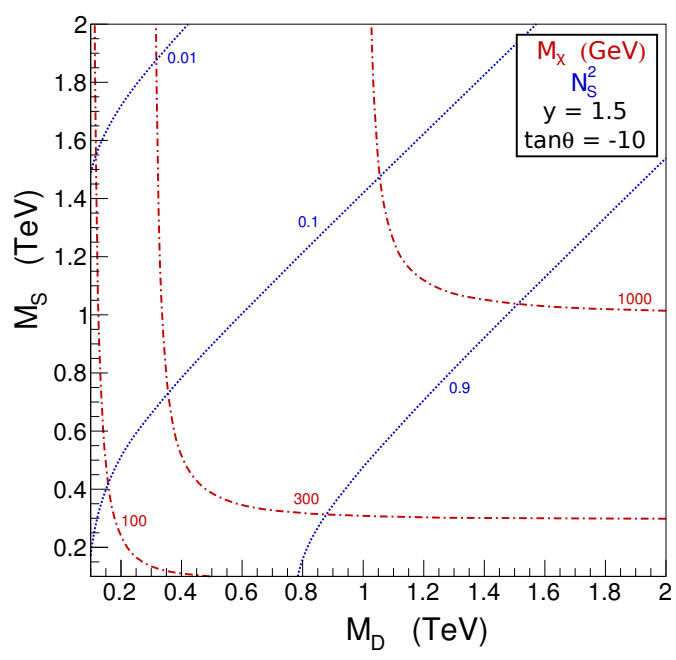

(d)

Figure 2. Mass and mixing angle contours in singlet-doublet fermion DM for $y=1.5$ and $\tan \theta=$ $\pm 2, \pm 10$. Contours shown are the same as in figure 1 .

Here we drop kinetic terms, along with all interactions involving only $S$ and $D$, which are DM self-interactions which do not effect DM annihilation or direct detection. For simplicity, we assume positive $M_{S}^{2}$ and $M_{D}^{2}$, although strictly speaking this is not necessary because the singlet and doublet acquire masses after electroweak symmetry breaking. Note that by applying a parity transformation $S \rightarrow-S$, we can flip the sign of $A$; consequently this sign is unphysical.

Scalar dark matter theories have a long pedigree. Scalar singlet DM is often considered the most minimal DM candidate, and has been studied extensively [55-60]. Current bounds on scalar singlet models with the correct relic density require $M_{S} \gtrsim 100 \mathrm{GeV}$ except for a small region of viable parameter space for $50 \mathrm{GeV} \lesssim M_{S} \lesssim 65 \mathrm{GeV}$ [61], but XENON1T reach expected to cover $M_{S} \gtrsim 10 \mathrm{TeV}$. Scalar doublet DM also has been studied extensively, most often in the case of a two-Higgs doublet model where only one Higgs receives a VEV [62], 
often called the "inert doublet model" [63]. Mixed singlet-doublet scalar models have also been considered previously [53, 64, 65], though most such studies have considered a sub-set of the possible phenomenology motivated by grand unification.

Paralleling the fermion case, singlet-doublet mixing for scalars produces three real neutral scalars and one charged scalar. Mixing among states is induced by the $A$ term after electroweak symmetry breaking. We can work in the basis of real neutral scalars, $\left(S, D_{R}, D_{I}\right)$, where $D_{R}$ and $D_{I}$ are the real and imaginary components of the neutral component of $D$. The mass squared matrix is

$$
\mathbf{M}^{2}=\left(\begin{array}{ccc}
M_{S}^{2}+\frac{1}{2} v^{2} \lambda_{S} & A v & 0 \\
A v & M_{D}^{2}+\frac{1}{2} v^{2}\left(\lambda_{D}+\lambda_{D}^{\prime}+\lambda_{D}^{\prime \prime}\right) & 0 \\
0 & 0 & M_{D}^{2}+\frac{1}{2} v^{2}\left(\lambda_{D}+\lambda_{D}^{\prime}-\lambda_{D}^{\prime \prime}\right)
\end{array}\right) .
$$

We assume the absence of CP violating couplings, so $D_{I}$ cannot mix with either $D_{R}$ or $S$. Focusing on mixed DM, we choose a very tiny but negative value of $\lambda_{D}^{\prime \prime}$ to ensure that the doublet which mixes has a smaller mass term. With this restriction we define the lightest mixed state as the DM particle $\chi=N_{S} S+N_{D} D_{R}$, where $N_{S}^{2}+N_{D}^{2}=1$.

DM mixing is induced by the upper left $2 \times 2$ block of the full mixing matrix, whose characteristic eigenvalue equation is

$$
0=\left(M_{\chi}^{2}-\tilde{M}_{S}^{2}\right)\left(M_{\chi}^{2}-\tilde{M}_{D}^{2}\right)-v^{2} A^{2},
$$

where $\tilde{M}_{S}^{2}=M_{S}^{2}+\frac{1}{2} v^{2} \lambda_{S}$ and $\tilde{M}_{D}^{2}=M_{D}^{2}+\frac{1}{2} v^{2}\left(\lambda_{D}+\lambda_{D}^{\prime}+\lambda_{D}^{\prime \prime}\right)$. The two eigenvalues of the mass-squared matrix are

$$
\frac{1}{2}\left[\tilde{M}_{S}^{2}+\tilde{M}_{D}^{2} \pm \sqrt{\left(\tilde{M}_{S}^{2}-\tilde{M}_{D}^{2}\right)^{2}+4 v^{2} A^{2}}\right]
$$

with the smaller eigenvalue corresponding to $M_{\chi}^{2}$. As shown earlier, the associated DM-Higgs coupling is given by the derivative of $M_{\chi}^{2}$ with respect to $v$,

$$
a_{h \chi \chi}=\frac{1}{2} v\left(\lambda_{S}+\lambda_{D}+\lambda_{D}^{\prime}+\lambda_{D}^{\prime \prime}\right)-\frac{2 v A^{2}+\frac{1}{2} v\left(\tilde{M}_{S}^{2}-\tilde{M}_{D}^{2}\right)\left(\lambda_{S}-\lambda_{D}-\lambda_{D}^{\prime}-\lambda_{D}^{\prime \prime}\right)}{\sqrt{\left(\tilde{M}_{S}^{2}-\tilde{M}_{D}^{2}\right)^{2}+4 v^{2} A^{2}}} .
$$

In our analysis we will make use of the simplifying limit $\lambda_{S}=\lambda_{D}=\lambda$ and $\lambda_{D}^{\prime}=\lambda_{D}^{\prime \prime}=0$. In this limit, $a_{h \chi \chi}$ simplifies to

$$
a_{h \chi \chi}=\lambda v-\frac{2 v A^{2}}{\sqrt{\left(M_{S}^{2}-M_{D}^{2}\right)^{2}+4 v^{2} A^{2}}} .
$$

In analogy with the case of fermion DM, we define the blind spot region by the condition $a_{h \chi \chi}=0$. However, in the scalar case it is complicated by the presence of Higgs couplings to the pure states. For fermionic DM, mixing is induced by Yukawa terms, leading to a correlation between mixing strength and the Higgs coupling. In the scalar case, however, for any degree of mixing, the direct quartic couplings can be modified to create a blind spot. The enhancement or suppression of $a_{h \chi \chi}$ depends on the sign of $\lambda$. For positive $\lambda$, a blind spot can occur, and interestingly, this is the sign preferred in general by considerations of 


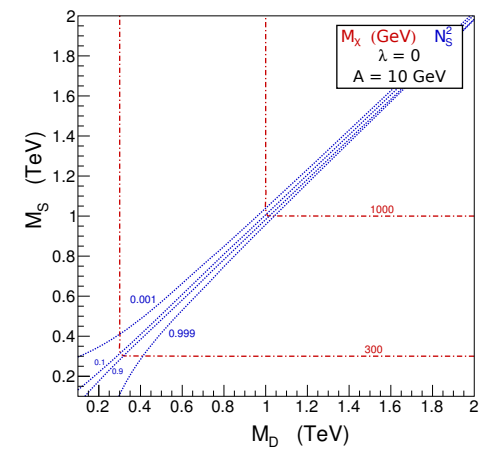

(a)

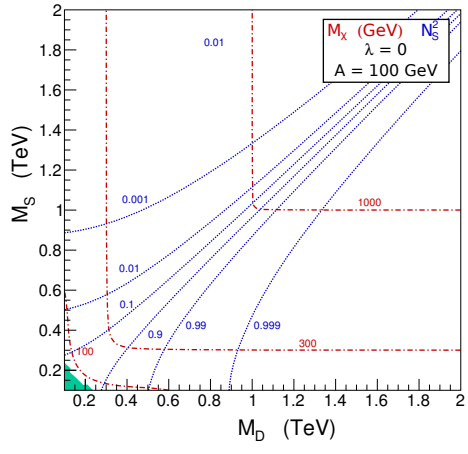

(b)

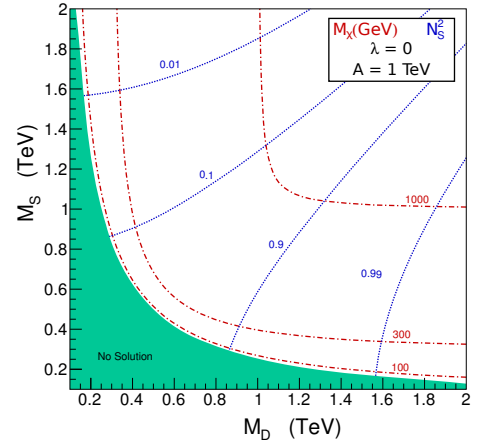

(c)

Figure 3. Mass and mixing angle contours for singlet-doublet scalar DM for $\lambda=0$ and $A=$ 10,100, $1000 \mathrm{GeV}$. Contours shown are the same as in figure 1.

tree-level vacuum stability. In particular, if $\lambda$ is too negative then the potential may contain unbounded from below directions at large field values.

As in the case of singlet-doublet fermion DM, we can characterize the amount of tuning required to accommodate a thermal relic abundance and evade direct detection. Again using eq. (2.5) and eq. (2.6), we define (using only the $2 \times 2$ mixing sub-matrix)

$$
\begin{aligned}
\xi_{\mathrm{WT}} & =\frac{\sqrt{\left(M_{S}^{2}-M_{D}^{2}\right)^{2}+4 v^{2} A^{2}}}{\left(M_{S}^{2}+M_{D}^{2}+\lambda v^{2}\right)} \\
\xi_{\mathrm{BS}} & =\frac{\left|\lambda v \sqrt{\left(M_{S}^{2}-M_{D}^{2}\right)^{2}+4 v^{2} A^{2}}-2 v A^{2}\right|}{|\lambda v| \sqrt{\left(M_{S}^{2}-M_{D}^{2}\right)^{2}+4 v^{2} A^{2}}+2 v A^{2}},
\end{aligned}
$$

to be the tuning measures for well-tempering and blind spot cancellations, respectively.

Last of all, we consider the DM mass and mixing angles in singlet-doublet scalar DM. Figure 3 shows contours of $M_{\chi}$ and $N_{S}^{2}$ in the $\left(M_{D}, M_{S}\right)$ plane, with $A=10,100,1000 \mathrm{GeV}$ and $\lambda_{S}=\lambda_{D}=\lambda_{D}^{\prime}=\lambda_{D}^{\prime \prime}=0$. For sufficiently small masses in the $A=100 \mathrm{GeV}$ and $A=1 \mathrm{TeV}$ cases, one of the eigenstates becomes tachyonic and thus the region is excluded. The well-mixed scenario occurs near $M_{S} \approx M_{D}$, with the degree of mixing dropping rapidly except for the very large value of $A=1 \mathrm{TeV}$. Likewise, except for $A=1 \mathrm{TeV}$ the mass contours are restricted to $M_{\chi} \approx M_{S}$ or $M_{\chi} \approx M_{D}$ except for very close to the $M_{S} \approx M_{D}$ line. Allowing for non-zero quartic DM-Higgs couplings does not qualitatively change the mass and mixing contours, but simply shifts their positions.

\subsection{Singlet-triplet scalar}

Lastly, we consider scalar DM comprised of a mixed singlet and triplet. For the sake of simplicity, we consider a real triplet, which necessarily carries zero hypercharge. The field content of this model is

\begin{tabular}{|c|c|c|}
\hline \multicolumn{3}{|c|}{ Model C } \\
\hline Field & Charges & Spin \\
\hline$S$ & $(\mathbf{1}, 0)$ & 0 \\
\hline$T$ & $(\mathbf{3}, 0)$ & 0 \\
\hline
\end{tabular}


with a corresponding Lagrangian,

$$
-\mathcal{L}_{\text {Model } C}=\frac{1}{2} M_{S}^{2} S^{2}+M_{T}^{2} \operatorname{tr}\left(T^{2}\right)+\frac{1}{2} \lambda_{S} S^{2}|H|^{2}+\lambda_{T} \operatorname{tr}\left(T^{2}\right)|H|^{2}+\kappa S H^{\dagger} T H,
$$

where once again we have dropped kinetic terms and interactions involving only $S$ and $T$. After electroweak symmetry breaking, the singlet and triplet mix via the dimensionless quartic interaction $\kappa$. Note that by applying the parity transformation, $S \rightarrow-S$, we can freely flip the sign of $\kappa$, so its sign is unphysical.

While models involving triplet scalar DM have not received the same level of attention as those with singlet or doublet scalar DM, both pure triplet $[21,66]$ and mixed singlettriplet $[67,68]$ have been considered. However, as for mixed singlet-doublet scalar DM, previous studies have primarily focused on a sub-set of parameter space motivated by grand unification.

The mass matrix for the singlet-triplet scalar case resembles the mixed sub-matrix for the singlet-doublet scalar, with the substitution $A \rightarrow-\kappa v / 4$ in the $(S, T)$ basis,

$$
\mathbf{M}^{2}=\left(\begin{array}{cc}
M_{S}^{2}+\frac{1}{2} v^{2} \lambda_{S} & -\frac{1}{4} \kappa v^{2} \\
-\frac{1}{4} \kappa v^{2} & M_{T}^{2}+\frac{1}{2} v^{2} \lambda_{T}
\end{array}\right) .
$$

After diagonalization, the DM particle is $\chi=N_{S} S+N_{T} T$, where $N_{S}^{2}+N_{T}^{2}=1$. Likewise, the mass-squared eigenvalues become

$$
\frac{1}{2}\left[\tilde{M}_{S}^{2}+\tilde{M}_{T}^{2} \pm \sqrt{\left(\tilde{M}_{S}^{2}-\tilde{M}_{T}^{2}\right)^{2}+\frac{1}{4} \kappa^{2} v^{4}}\right],
$$

where $\tilde{M}_{S}^{2}=M_{S}^{2}+\lambda_{S} v^{2} / 2$ and $\tilde{M}_{T}^{2}=M_{T}^{2}+\lambda_{T} v^{2} / 2$. However, due to the $v$-dependence of the mixing term for the singlet-triplet case, the same substitution does not apply to $a_{h \chi \chi}$, which has the form

$$
a_{h \chi \chi}=\frac{1}{2} v\left(\lambda_{S}+\lambda_{T}\right)-\frac{\frac{1}{4} \kappa^{2} v^{3}+\frac{1}{2} v\left(\tilde{M}_{S}^{2}-\tilde{M}_{T}^{2}\right)\left(\lambda_{S}-\lambda_{T}\right)}{\sqrt{\left(\tilde{M}_{S}^{2}-\tilde{M}_{T}^{2}\right)^{2}+\frac{1}{4} \kappa^{2} v^{4}}} .
$$

The contribution to the Higgs coupling from the mixing term in the singlet-triplet case is roughly twice as large relative to the singlet-doublet case for models with an equivalent mass spectrum and mixing. For $\lambda_{S}=\lambda_{D}=\lambda$, this reduces to

$$
a_{h \chi \chi}=\lambda v-\frac{\frac{1}{4} \kappa^{2} v^{3}}{\sqrt{\left(M_{S}^{2}-M_{T}^{2}\right)^{2}+\frac{1}{4} \kappa^{2} v^{4}}} .
$$

As in the singlet-doublet case, cancellations in the DM-Higgs coupling only occur if $\lambda$ is positive, with the same implication that vacuum stability favors positive values of $\lambda$ and thus cancellation. From eq. (2.5) and eq. (2.6), we define tuning measures for blind spot cancellations and well-tempering,

$$
\begin{aligned}
\xi_{\mathrm{WT}} & =\frac{\sqrt{\left(M_{T}^{2}-M_{D}^{2}\right)^{2}+\frac{1}{4} \kappa^{2} v^{4}}}{\left(M_{S}^{2}+M_{T}^{2}+\lambda v^{2}\right)} \\
\xi_{\mathrm{BS}} & =\frac{\left|\lambda v \sqrt{\left(M_{S}^{2}-M_{T}^{2}\right)^{2}+\frac{1}{4} \kappa^{2} v^{2}}-\frac{1}{4} \kappa^{2} v^{3}\right|}{|\lambda v| \sqrt{\left(M_{S}^{2}-M_{T}^{2}\right)^{2}+\frac{1}{4} \kappa^{2} v^{2}}+\frac{1}{4} \kappa^{2} v^{3}} .
\end{aligned}
$$




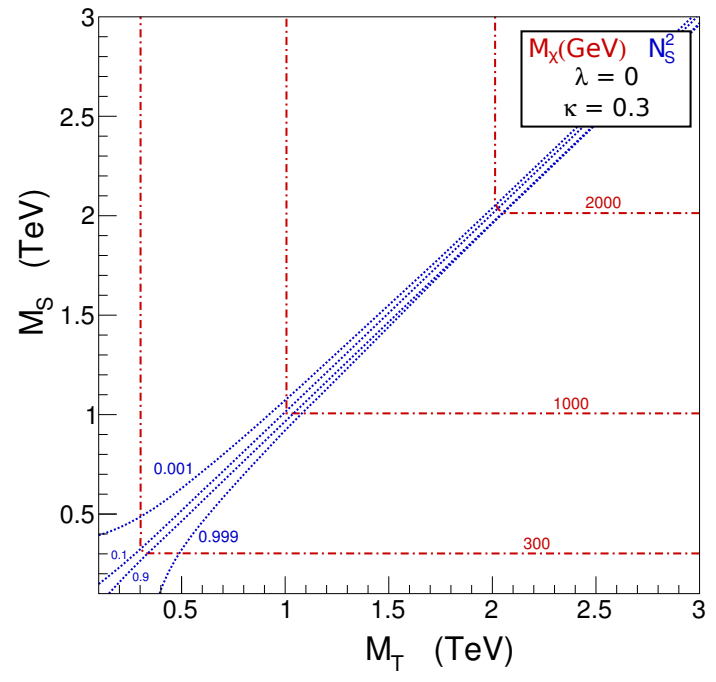

(a)

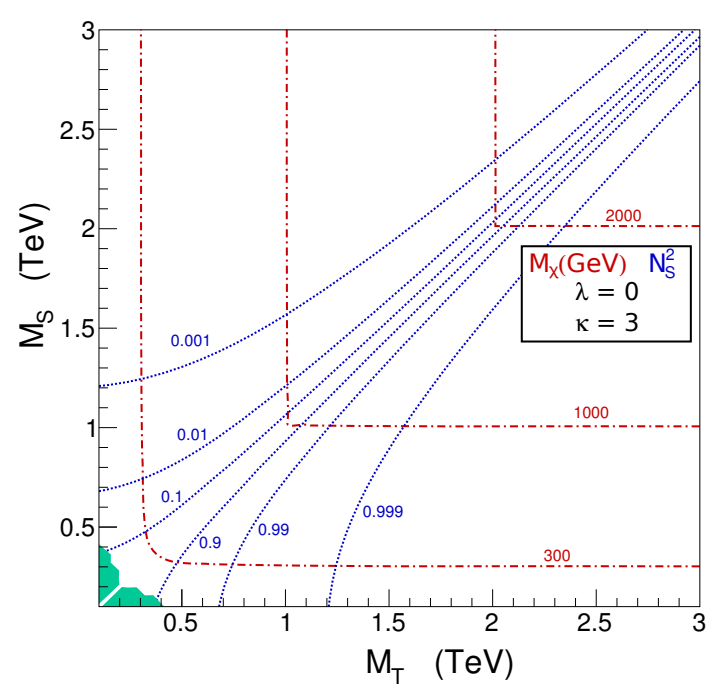

(b)

Figure 4. Mass and mixing angle contours for singlet-triplet scalar DM for $\lambda=0$ and $\kappa=0.3,3$. Contours shown are the same as in figure 1.

As before, we plot the DM mass, $M_{\chi}$, and singlet mixing angle squared, $N_{S}^{2}$, as a function of the parameter space in figure 4. Qualitatively, our results are similar to the singlet-doublet case. However, there is a major quantitative difference, which is that mixing effects are minimal away from $M_{S} \approx M_{D}$ even at very large values of the coupling, $\kappa=3$. As we will see in section 5 , this implies substantially different experimental constraints on singlet-doublet versus singlet-triplet scalar DM.

\section{Model A: singlet-doublet fermion DM}

To begin, we will analyze the full four dimensional parameter space of singlet-doublet fermion DM, $\left(M_{S}, M_{D}, y_{D_{1}}, y_{D_{2}}\right)$, imposing no constraints beyond the definition of the theory in section 2.1. We will display experimental constraints and regions consistent with $\Omega_{\chi}=\Omega_{\mathrm{DM}}$ as a function of the bare masses $M_{S}$ and $M_{D}$, fixing $(y, \theta)$ to several characteristic values. We will then focus on the subspace of thermal relic DM, fixing one of the model parameters $\left(M_{D}, M_{S}\right.$, or $\theta$, depending on the plot) to accommodate $\Omega_{\chi}=\Omega_{\mathrm{DM}}$. Lastly, we will study the thermal relic scenario further restricted to the parameter space residing exactly at the present (and future) limits of direct detection experiments. In particular, for this analysis we will fix $y$ to $\sigma^{\mathrm{SI}}=\sigma_{\mathrm{LUX}}^{\mathrm{SI}}$ or $\sigma^{\mathrm{SI}}=\sigma_{\mathrm{X} 1 \mathrm{~T}}^{\mathrm{SI}}$, corresponding to the space of models which are "marginally excluded" by LUX or XENON1T, respectively.

\subsection{Exclusion plots (general)}

First, we consider the unconstrained parameter space, focusing on the position of the $\Omega_{\chi}=\Omega_{\mathrm{DM}}$ line. A pure singlet Majorana fermion does not couple to the SM at the renormalizable level, so its thermal relic abundance is typically very large. Meanwhile, a pure doublet has $\Omega_{\chi}=\Omega_{\mathrm{DM}}$ for $M_{D} \simeq 1 \mathrm{TeV}$ [69], with $\Omega_{\chi}>\Omega_{\mathrm{DM}}$ for $M_{D} \gtrsim 1 \mathrm{TeV}$ and $\Omega_{\chi}<\Omega_{\mathrm{DM}}$ for $M_{D} \lesssim 1 \mathrm{TeV}$. Annihilation of the DM into gauge bosons occurs entirely through the electroweak charged component of the DM. Hence, this contribution to the annihilation 
cross-section is suppressed by the doublet mixing angles, $N_{D_{1}}$ and $N_{D_{2}}$. In principle, annihilation can also occur via Higgs exchange, and this will be enhanced at large $y$. However, annihilation via the Higgs is a p-wave suppressed process for Majorana fermions, and thus sub-dominant to gauge boson processes unless $y$ is very large.

In figure 5, we have plotted constraints in the $\left(M_{D}, M_{S}\right)$ plane, fixing $y=0.3$ and $\tan \theta= \pm 2$ (top) or \pm 10 (bottom). For $M_{S} \lesssim 1 \mathrm{TeV}$, significant well-tempering of the DM mixing angles is required to produce $\Omega_{\chi}=\Omega_{\mathrm{DM}}$ for all parameter combinations. This is the case because $y v \ll M_{S, D}$, requiring $M_{S} \approx M_{D}$ for any significant level of mixing. For $M_{S} \gtrsim 1 \mathrm{TeV}$ the thermal relic line asymptotes to $M_{D} \simeq 1 \mathrm{TeV}$, the mass at which pure doublet $\mathrm{DM}$ is a thermal relic with the correct abundance. The fact that $\Omega_{\chi}=\Omega_{\mathrm{DM}}$ is not possible for $M_{D}>1 \mathrm{TeV}$ implies that the Higgs coupling $y=0.3$ affects the annihilation cross-section primarily through mixing angles that control gauge boson processes. That is, processes involving the Higgs boson directly do not strongly affect the DM annihilation cross-section.

In terms of direct detection, the value of $\sigma^{\mathrm{SI}}$ depends sensitively on the $\operatorname{sign}$ of $\tan \theta$. For $\tan \theta>0$ it lies in the range $0.01 \mathrm{zb} \lesssim \sigma^{\mathrm{SI}} \lesssim 10 \mathrm{zb}$ throughout most of the region shown, and exhibits no blind spot behavior. Conversely, for $\tan \theta<0$, the maximum value of $\sigma^{\mathrm{SI}}$ is reduced, and a blind spot occurs where $\sigma^{\mathrm{SI}}$ vanishes (the minimum value shown is $0.001 \mathrm{zb}$ ). The position of the blind spot changes for different values of $\tan \theta$, and is located at $M_{S}+M_{D} \sin 2 \theta \approx 0$, roughly consistent with the blind spot condition of eq. (2.13).

For positive $\tan \theta$, the thermal line is constrained by LUX for $M_{S} \gtrsim 1.1 \mathrm{TeV}$ for $\tan \theta=2$ and $M_{S} \gtrsim 1 \mathrm{TeV}$ for $\tan \theta=10$. For negative $\tan \theta$, however, LUX provides no bound for $\tan \theta=-2$ and bounds the thermal scenario only up to $900 \mathrm{GeV}$ for $\tan \theta=-10$. Meanwhile, for both values of $\tan \theta$, XENON1T constrains the thermal scenario well into the nearly pure doublet region, as well as large swaths of non-thermal scenarios. Even after XENON1T, large swaths of parameter space will still be allowed at small and negative $\tan \theta$, and for $\tan \theta=-2$ a small portion of the thermal line with $M_{S} \sim 200 \mathrm{GeV}$ remains viable. The relatively low values of $\sigma^{\mathrm{SI}}$ and weak exclusion for $\tan \theta=-2$ are due to a blind spot near $\tan \theta=-1$ for doublet-like DM [49]. At large values of $|\tan \theta|$ (corresponding to large $\tan \beta$ in the MSSM), the $\operatorname{sign}$ of $\tan \theta$ becomes unphysical [49], as shown by the relative similarity of the contours for $\tan \beta= \pm 10$ as opposed to $\tan \theta= \pm 2$ in figure 5 .

There are two major implications of figure 5. First, the region consistent with a thermal relic is already quite constrained by LUX for $y=0.3$. XENON1T will further constrain this region, excluding at least up to the point at which DM is nearly a pure doublet except for a very finely tuned region of parameter space. Second, the sensitivity of limits depends greatly on the sign of $\tan \theta$, due to blind spot cancellation points, and in small regions of parameter space even relatively light masses of a few hundred $\mathrm{GeV}$ remain viable.

As shown in figure 6, the situation changes drastically when the Higgs-DM coupling is increased to $y=1.5$. There is a significant shift in the position of the thermal relic line, primarily toward larger values of $M_{D}$ for a given value of $M_{S}$. This behavior stems from the increase in the size of the off-diagonal terms in the mass matrix, resulting in $y v \sim M_{S, D}$ for a larger portion of the space scanned in the figures. As a result, well-tempering is no longer required to produce significant mixing, and so $M_{D}$ must be increased significantly to suppress DM annihilation mediated through the doublet component. Moreover, a significant portion of the $\Omega_{\chi}=\Omega_{\mathrm{DM}}$ line is located at $M_{D}>1 \mathrm{TeV}$, implying that annihilation for large $y$ is substantially stronger than for a pure doublet. The dominant effect is enhanced annihilation mediated by a $Z$-boson due to a modification of the $\chi-Z$ coupling, $i c_{Z \chi \chi} \bar{\chi} \gamma^{\mu} \gamma^{5} \chi Z_{\mu}$. For 


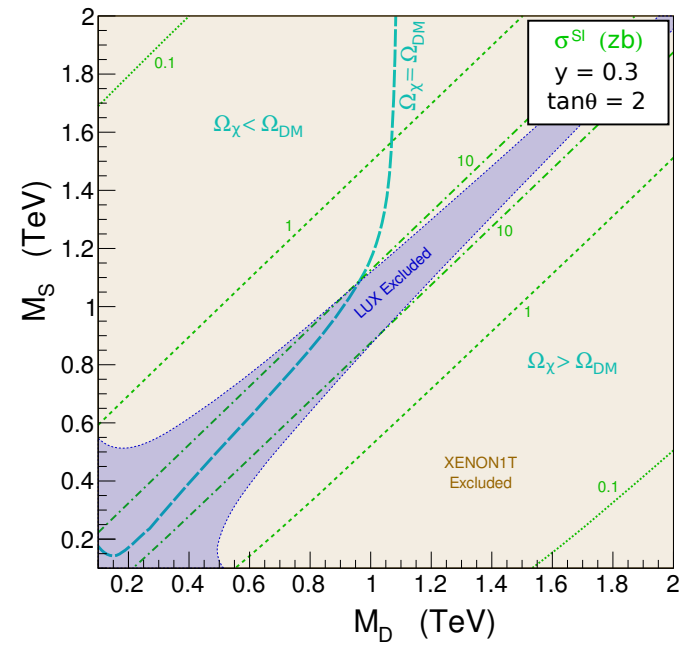

(a)

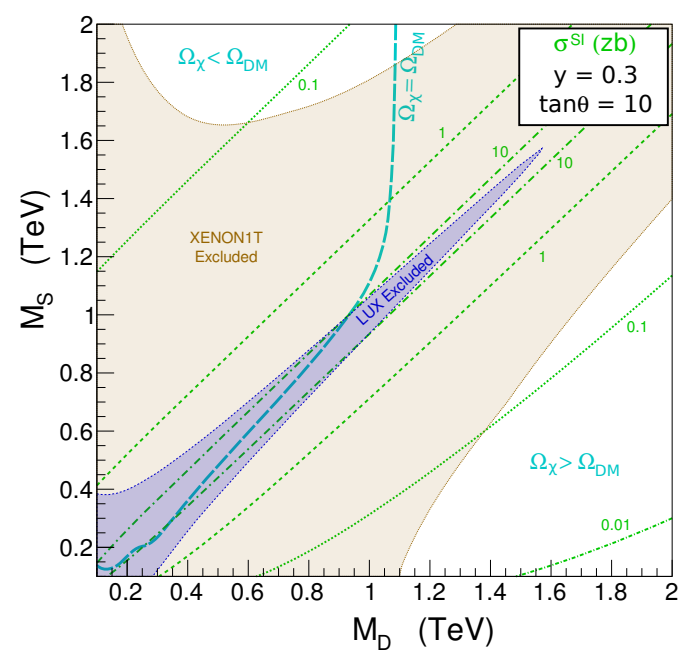

(c)

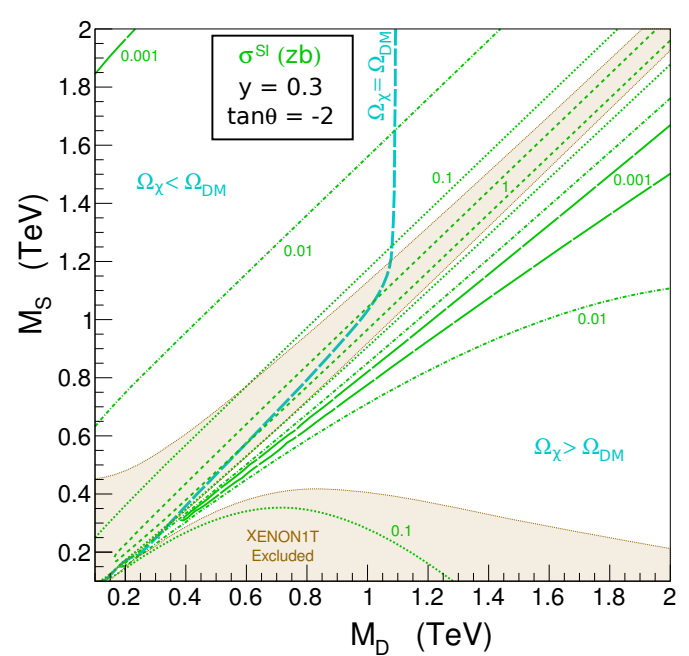

(b)

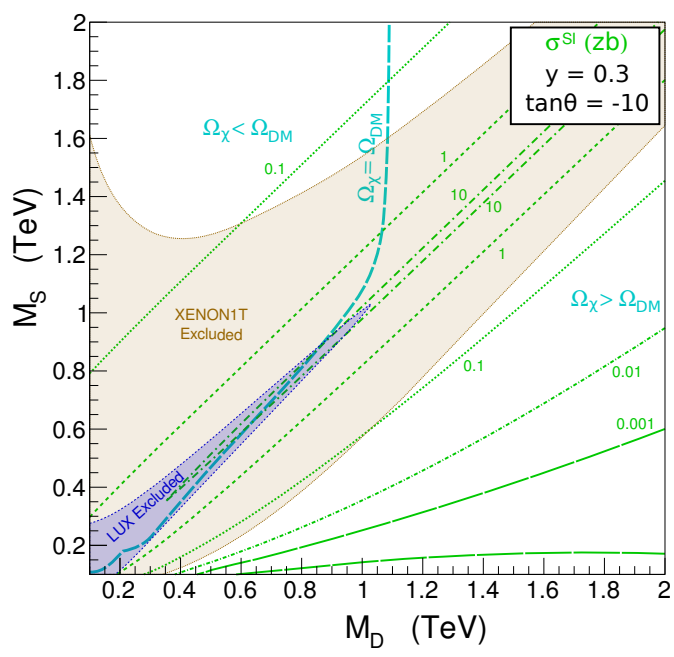

(d)

Figure 5. Direct detection prospects for $y=0.3$ and $\tan \theta= \pm 2, \pm 10$. Shown are contours of $\sigma^{\mathrm{SI}}$ in zb (green, various styles) together with current bounds from LUX (blue shaded) and projected reach at XENON1T (gold shaded). Away from the line consistent with the observed relic density (teal dashed), we compute LUX and XENON1T bounds assuming that the DM relic density is equal to the observed one due to a non-thermal cosmology.

mixed scenarios

$$
c_{Z \chi \chi}=\frac{g}{2 \cos \theta_{W}}\left(N_{D_{1}}^{2}-N_{D_{2}}^{2}\right)
$$

where $g$ is the weak gauge coupling and $\theta_{W}$ is the Weinberg angle. In the $y \rightarrow 0$ limit, the singlet and doublet partially decouple, resulting in $N_{D_{1}}=N_{D_{2}} \rightarrow 0$ for $M_{D}>M_{S}$ or $\left|N_{D_{1}}\right|=\left|N_{D_{2}}\right| \rightarrow 1 / \sqrt{2}$ for $M_{D}<M_{S}$. In either case, $c_{Z \chi \chi} \rightarrow 0$, so long as the very small splitting necessary to break the doublet into two Majorana states remains. However, for larger values of $y$ and $\tan \theta \neq \pm 1$, the degree of $S-D_{1}$ mixing and $S-D_{2}$ mixing is different, resulting in $\left|N_{D_{1}}\right| \neq\left|N_{D_{2}}\right|$ and thus $c_{Z \chi \chi} \neq 0$. The size of $c_{Z \chi \chi}$ grows with the 


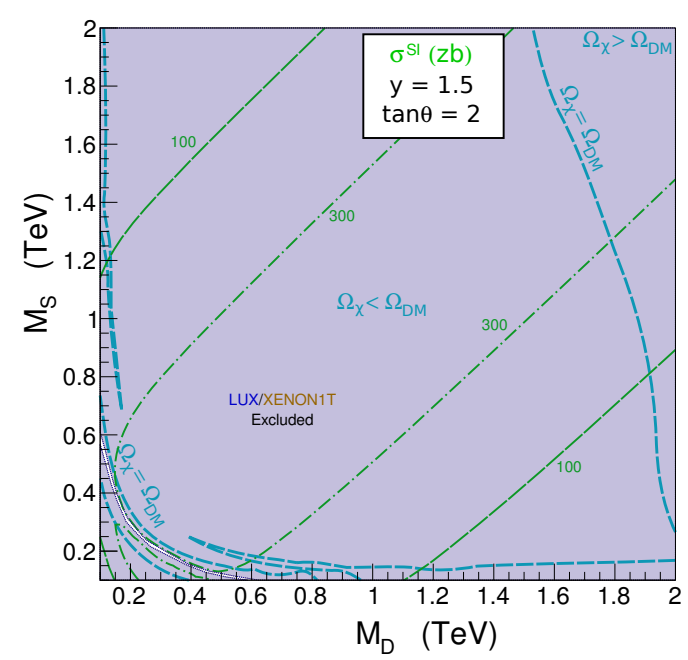

(a)

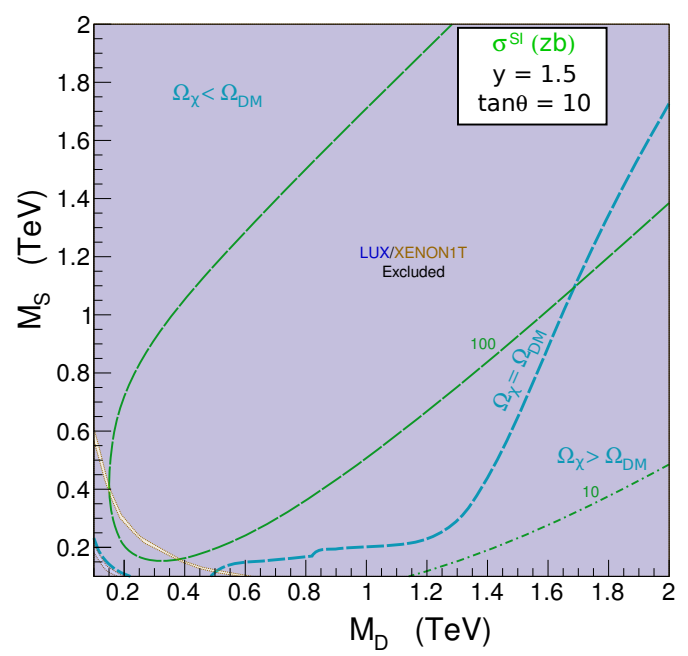

(c)

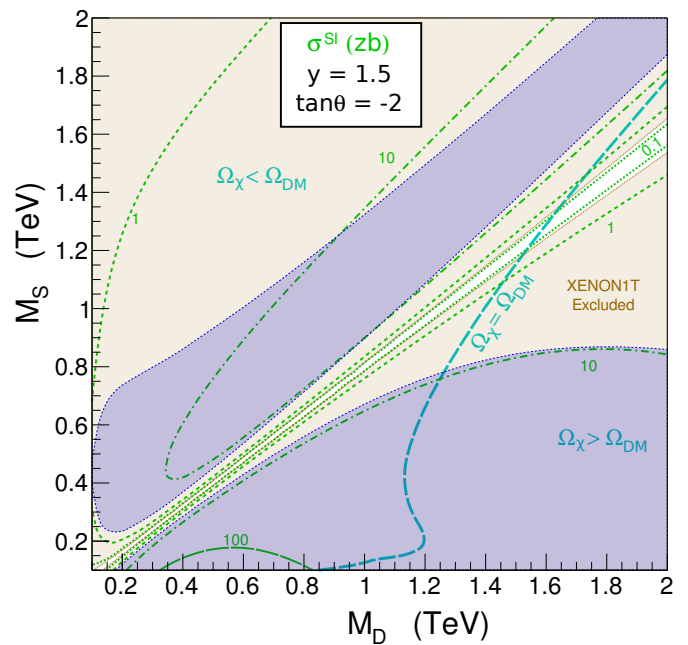

(b)

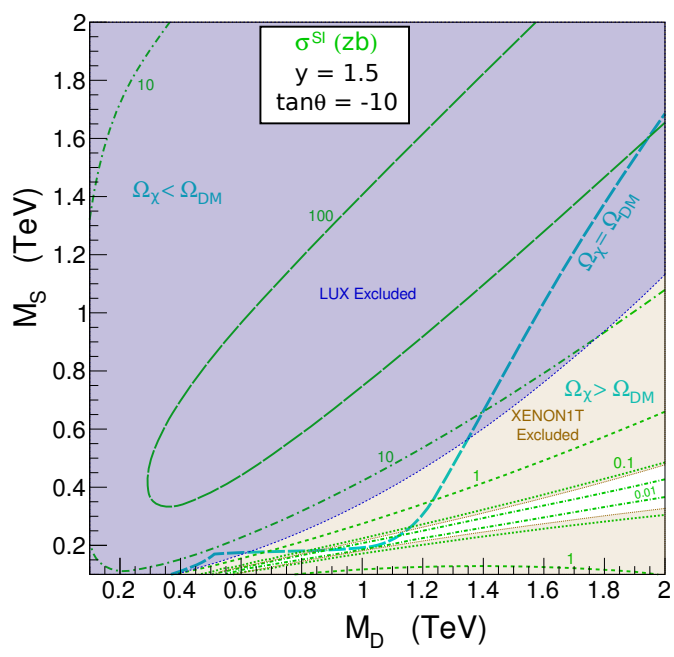

(d)

Figure 6. Direct detection prospects for $y=1.5$ and $\tan \theta= \pm 2, \pm 10$. Contours shown are the same as in figure 5.

degree of mixing, and thus with $y$, though it of course can never exceed $g / 2 \cos \theta_{W}$. For large $y$, enhanced Higgs-mediated annihilation also becomes important, though such processes remain p-wave suppressed and are somewhat smaller than the $Z$-mediated diagrams.

While stronger annihilation channels shifts the $\Omega_{\chi}=\Omega_{\mathrm{DM}}$ line to larger values of $M_{D}>$ $1 \mathrm{TeV}$ over most of the range shown, for sufficiently large $M_{S} \sim 3-4 \mathrm{TeV}$, the thermal relic line asymptotes to the $M_{D} \approx 1 \mathrm{TeV}$ line corresponding to nearly-pure doublet DM. In the right panels, $\tan \theta<0$ and $c_{h \chi \chi}$ is suppressed, so $Z$-mediated processes dominate annihilation. As a result, annihilation becomes markedly stronger approaching the $M_{S} \approx M_{D}$ line, and the $\Omega_{\chi}=\Omega_{\mathrm{DM}}$ line moves to larger $M_{D}$ with increasing $M_{S}$. The coupling $c_{Z \chi \chi}$ is marginally smaller for $\tan \theta=-2$ than for $\tan \theta=-10$, but not sufficiently so to produce significantly different results. For $\tan \theta>0$, however, the $\Omega_{\chi}=\Omega_{\mathrm{DM}}$ line shows distinctly different behavior for $\tan \theta=2$. Because $Z$-mediated processes vanish identically in the limit 
of $c_{Z \chi \chi} \rightarrow 0$ for $\tan \theta \rightarrow \pm 1$, this coupling is suppressed in the neighborhood of $\tan \theta \approx 1$. As a result, the $\Omega_{\chi}=\Omega_{\mathrm{DM}}$ contour is located at a lower value of $M_{D}$ for $\tan \theta=2$. Higgs-mediated annihilation compensates somewhat at lower $M_{S}$, and indeed the $\tan \theta=2$ case has the largest Higgs coupling of the cases shown, but Higgs-mediated annihilation does not exhibit the same degree of enhancement for $M_{D} \approx M_{S}$. As a result, the $\Omega_{\chi}=\Omega_{\mathrm{DM}}$ line is located at large $M_{D}$ for small $M_{S}$ and shifts to smaller values of $M_{D}$ as $M_{S}$ increases. The $\tan \theta=10$ case, however, is far enough from $\tan \theta=1$ that the behavior seen in $\tan \theta<0$ is recovered.

Moreover, the availability of annihilation channels is important for low masses - in the lower two panels of figure 6 , the $\Omega_{\chi}=\Omega_{\mathrm{DM}}$ line is nearly horizontal over a range of several hundred $\mathrm{GeV}$ for $M_{D}$ in multiple plots for $M_{S} \sim m_{t}$. The increase of $M_{D}$ for nearly fixed $M_{S}$ in this region effectively reduces the mixing angle to compensate for an enhancement in annihilation from the opening of the $\chi \chi \rightarrow t \bar{t}$ annihilation channel. Likewise, the singletdoublet mixing terms are so large that $M_{\chi} \rightarrow 0$ for $\tan \theta>0$ and $M_{S, D}$ of order a few hundred GeV. For $\tan \theta<0$ this does not occur due to the structure of the mass matrix. In the upper left panel of figure $6, y=1.5, \tan \theta=2$ and the $\Omega_{\chi}=\Omega_{\mathrm{DM}}$ line is roughly parallel to the low mass DM contours. This occurs because the relic abundance is strongly controlled by the opening of annihilation channels when the DM mass crosses the bottom quark, $W$ boson, and top quark thresholds.

Regarding direct detection, for $y=1.5$, the raw value of $\sigma^{\mathrm{SI}}$ is increased by more than an order of magnitude relative to $y=0.3$. LUX excludes the entire region shown for $\tan \theta>0$, except for a small region at low $M_{S, D}$ for which $M_{\chi} \lesssim m_{b}$ is below the experimental threshold. However, this low mass region retains a large Higgs coupling and will be excluded by constraints on the invisible decay width of the Higgs [70]. Blind spot cancellations occur for $\tan \theta<0$, however, with large portions of the thermal relic line remaining viable given LUX limits. XENON1T still has much greater reach for $\tan \theta<0$, with the increased Higgs coupling resulting in only the blind spot and an associated small portion of the thermal relic line evading XENON1T sensitivity.

\subsection{Exclusion plots (thermal relic)}

Next, we consider singlet-doublet fermion DM in a reduced parameter space. We focus on thermal relic DM by restricting to regions that saturate the observed DM abundance, defined by $\Omega_{\chi}=\Omega_{\mathrm{DM}}$. Concretely, we fix one of parameters $\left(M_{S}, M_{D}, y, \theta\right)$ to saturate the thermal relic constraint.

Figure 7 depicts constraints in the $\left(y, M_{S}\right)$ plane, fixing $\tan \theta$ to various values and setting $M_{D}$ so that $\Omega_{\chi}=\Omega_{\mathrm{DM}}$. As before, the $\operatorname{sign}$ of $\tan \theta$ has a significant effect on DM properties. For $\tan \theta>0$ there are no blind spots, and for $y \lesssim 0.5$, the cross-section $\sigma^{\text {SI }}$ grows almost monotonically with $y$ and decreases with $M_{S} \sim M_{\chi}$. In this region, DM annihilation results primarily from gauge interactions typical to nearly-pure doublet DM. For $y \gtrsim 0.5$, however, Higgs-mediated and $Z$-mediated diagrams become important, with their relative contributions increasing with $y$. As a result, $\sigma^{\text {SI }}$ actually decreases for increasing $y$ for $y \gtrsim 1$, due to a reduction in the mixing to limit the Higgs- and $Z$-mediated annihilation processes that increase with $y$. A small region of larger $\sigma^{\mathrm{SI}}>100 \mathrm{zb}$ is also present for $M_{S} \lesssim 200 \mathrm{GeV}$ in the $\tan \theta=2$ case, associated with large splitting which drives $M_{\chi}<m_{W}$ and thus requires a larger Higgs coupling to produce appropriate levels of annihilation. Figure 7 also shows that direct detection limits from LUX bound $y \lesssim 0.3$ for $M_{S} \lesssim 1 \mathrm{TeV}$ in the $\tan \theta>0$ case, while XENON1T reach covers all regions except for extremely small $y$ for any $M_{S} \lesssim 1 \mathrm{TeV}$ for $\tan \theta$, and even up to $M_{S}=2 \mathrm{TeV}$ XENON1T limits $y \lesssim 0.3$. 


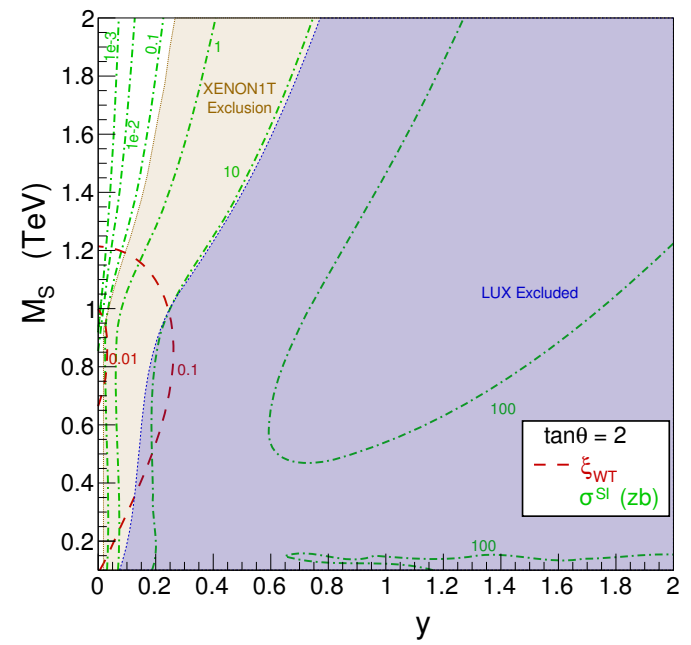

(a)

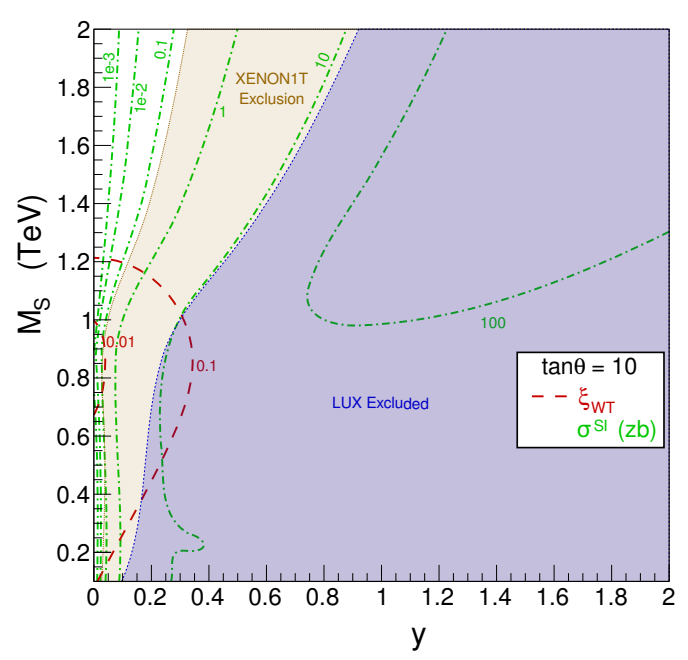

(c)

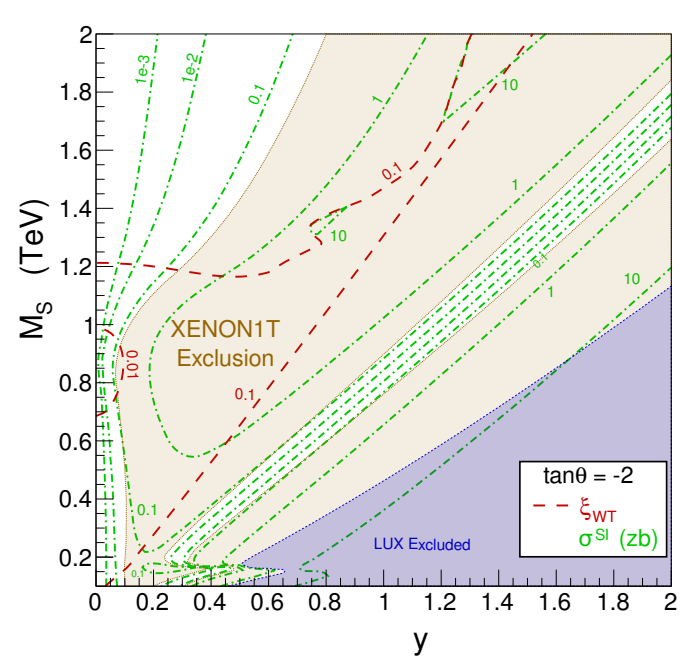

(b)

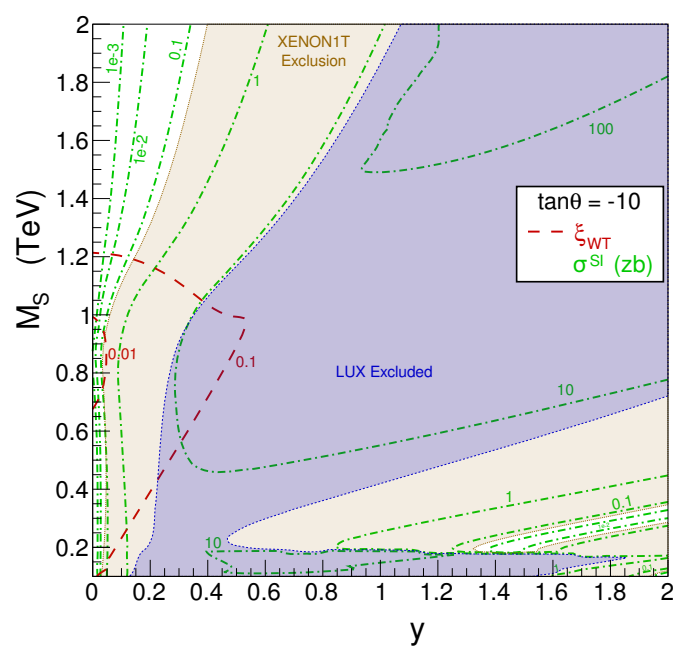

(d)

Figure 7. Well-tempering and direct detection prospects for fixed $\Omega_{\chi}=\Omega_{D M}$ as a function of $y$ and $M_{S}$. Shown are the well-tempering measure $\xi_{\mathrm{WT}}$ (red dashed) and direct detection cross-section $\sigma^{\mathrm{SI}}$ in $\mathrm{zb}$ (green, various styles). Also shown are the regions currently excluded by LUX (blue shaded) and the projected reach of XENON1T (gold shaded).

For $\tan \theta<0$, both the general suppression in the $c_{h \chi \chi}$ and the existence of blind spots significantly affect the results. The shape of the $\sigma^{\text {SI }}$ contours for small $y$ is similar to the $\tan \theta>0$ case, but the values are significantly reduced. For $\tan \theta=-2$ in particular this suppression is sufficient that no LUX exclusion exists for $y \lesssim 0.5$ even for small $M_{S}$, and only bounds $M_{S} \gtrsim 1 \mathrm{TeV}$ even for $y=2$. Even with this suppression, however, XENON1T still has strong constraining power down to small values of $y$ for $M_{S} \lesssim 1 \mathrm{TeV}$. The position of blind spots which also avoid XENON1T projected bounds is set by $M_{D}$, and they occur primarily at larger values of $y$ since such values are required to produce sufficient splitting between $M_{S}$ and $M_{D}$ for the values of $\tan \theta$ shown. These blind spots are more difficult to accommodate at large $\tan \theta$, where they require a larger hierarchy between $M_{\chi}$ and $M_{D}$. 


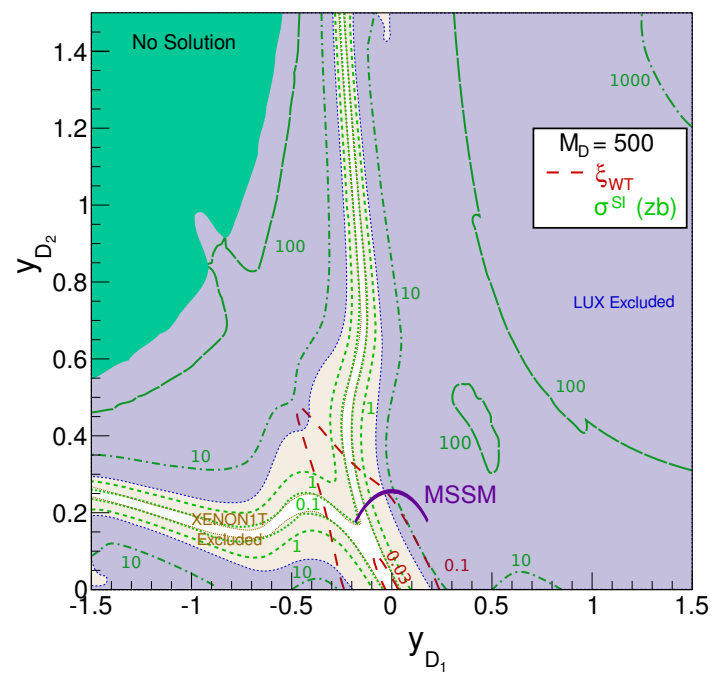

(a)

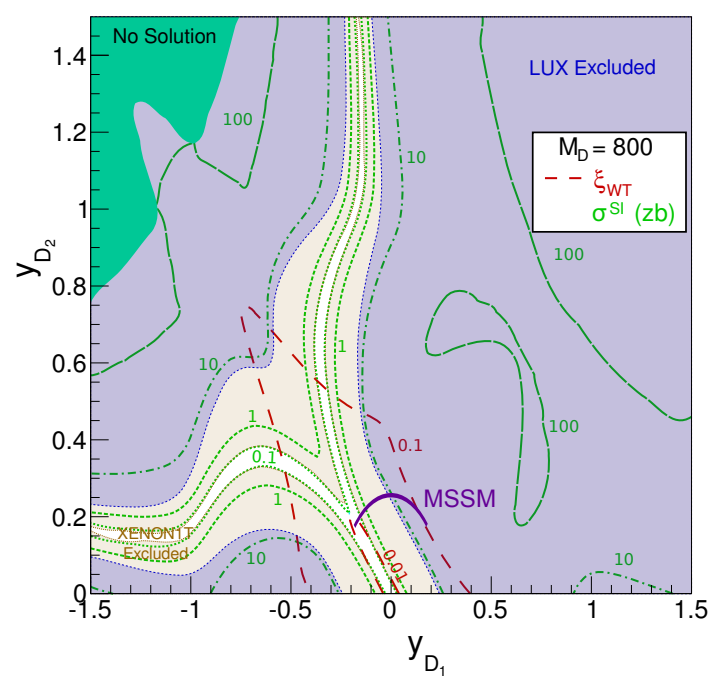

(b)

Figure 8. Well-tempering and direct detection prospects for fixed $\Omega_{\chi}=\Omega_{\mathrm{DM}}$ as a function of $y_{D_{1}}$ and $y_{D_{2}}$. Contours shown are the same as in figure 7 .

These plots also depict the well-tempering parameter, $\xi_{\mathrm{WT}}$, which characterizes the level of degeneracy required among the mixed neutral states in order to yield the correct relic abundance. The existing LUX bound still allows for thermal relics with moderate well-tempering, $\xi_{\mathrm{WT}} \sim 0.1$, especially for $\tan \theta<0$. It also places moderate limits on $y \lesssim 0.3$ for $\tan \theta>0$, and allows larger values of $y$ for $\tan \theta<0$. However, XENON1T will strongly alter the available parameter space of this simplified model. Projected limits from XENON1T typically constrain $\xi_{\mathrm{WT}} \lesssim 0.1$ throughout for $M_{\chi} \lesssim 1 \mathrm{TeV}$, with $\xi_{\mathrm{WT}} \lesssim 10^{-2}$ for $700 \mathrm{GeV} \lesssim M_{\chi} \lesssim 1 \mathrm{TeV}$, which is a substantial degree of tuning. A notable exception is exactly on blind spots, where there is no direct detection limit; however, residing on these cancellation regions constitutes an additional tuning.

Figure 8 depicts direct detection constraints in the thermal relic parameter space, only shown in the $\left(y_{D_{1}}, y_{D_{2}}\right)$ plane at fixed $M_{D}$, and fixing $M_{S}$ to satisfy the relic constraint. For certain regions within this plane there are multiple solutions for $\Omega_{\chi}=\Omega_{\mathrm{DM}}$; in such cases the solution with the largest value of $M_{\chi}$ is displayed. ${ }^{1}$ Here $\tan \theta=y_{D_{2}} / y_{D_{1}}$ is positive (negative) in the right (left) quadrant. As in figure $7, \xi_{\mathrm{WT}}$ is small for small $y$ and grows as $y$ becomes large. However, in contrast with figure 7 where $M_{D}$ was allowed to grow to offset the increasing value of $y v$, in figure $8 M_{D}<1 \mathrm{TeV}$ throughout. As such, some mixing is still required to dilute the annihilation strength, so $M_{S}$ is never even approximately decoupled. For $\tan \theta>0\left(y_{D_{1}}>0\right)$ the large $y$ region induces a significant mass splitting, and is thus dominated by small values of $M_{\chi} \lesssim m_{t}$ and correspondingly large $\sigma^{\mathrm{SI}}$, closing annihilation channels and reducing coannihilation to compensate for enhanced annihilation strength. For $\tan \theta<0\left(y_{D_{1}}<0\right)$ the induced mass splitting is significantly smaller, particularly for $\tan \theta \approx-1$, and so no such low mass solutions exist. This produces a region for which $\Omega_{\chi}=\Omega_{\mathrm{DM}}$ is unachievable by varying $M_{S}$ alone. The size of both the small mass and no solution regions decreases with increasing $M_{D}$.

\footnotetext{
${ }^{1}$ Secondary solutions for $\tan \theta>0$ tend to be at a lower mass and thus more strongly constrained by direct detection; secondary solutions for $\tan \theta<0$ have similar masses and $\sigma^{\text {SI }}$ to the primary solutions.
} 


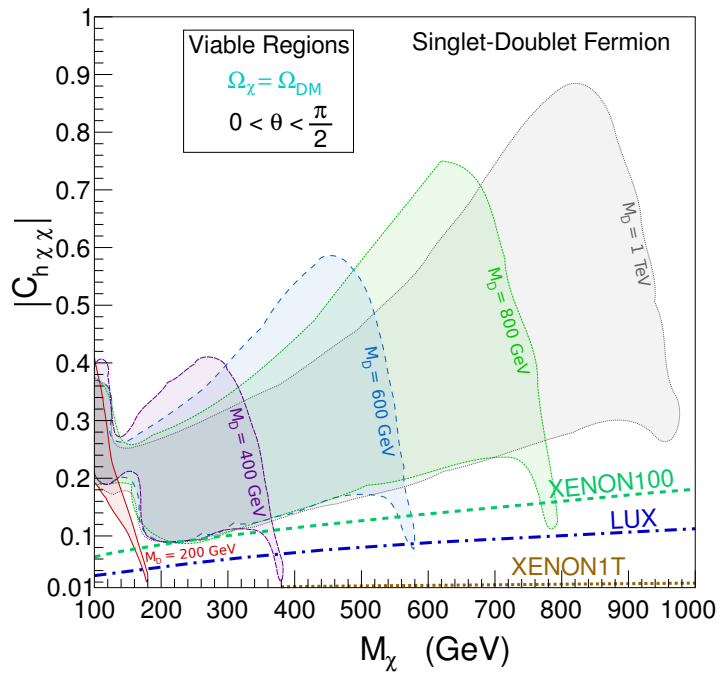

(a)

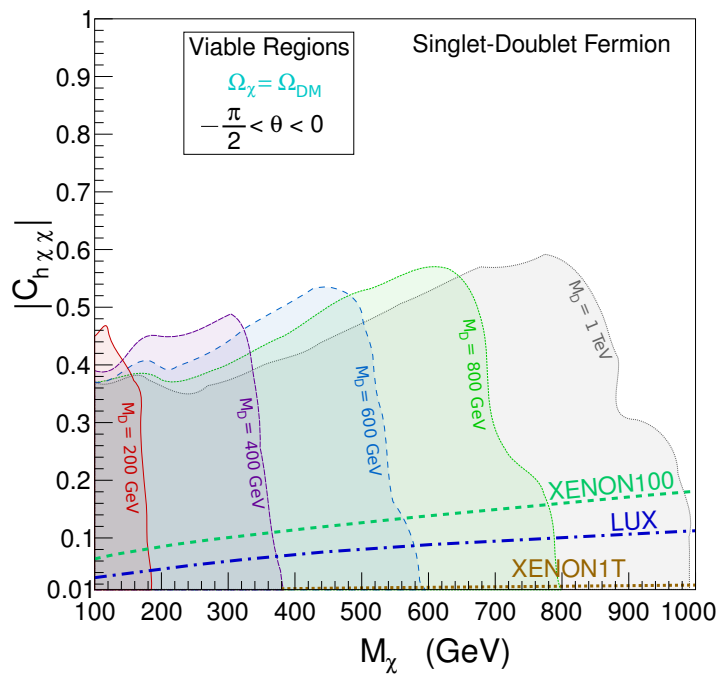

(b)

Figure 9. Viable regions with $\Omega_{\chi}=\Omega_{\mathrm{DM}}$ as a function of $M_{\chi}$ and $c_{h \chi \chi}$. Regions are shown for various values of $M_{D}$ and for the ranges $0<\theta<\pi / 2$ (left) and $-\pi / 2<\theta<0$ (right). Also shown are upper limits on $\left|c_{h \chi \chi}\right|$ allowed by XENON100 (teal dashed), LUX (blue dot-dashed) and projected upper limits from XENON1T (gold dashed).

At present, LUX strongly constrains this simplified model for $y_{D_{1}}>0$, only allowing a small region at small $y$; however, once again a blind spot exists for $y_{D_{1}}<0$ which leaves open large swaths of available parameter space. This blind spot extends to arbitrarily large values of $y$ (limited, of course, by perturbativity), though the trajectory varies with mass at large $y$. However, after XENON1T, only models very close to this blind spot will still be viable. Regarding well-tempering, for $y_{D_{1}}>0$, only a very small value of $y$ will be allowed after XENON1T, corresponding to a significant degree of well-tempering, $\xi_{\mathrm{WT}} \lesssim 0.03$. For $y_{D_{1}}<0$, well-tempering is still substantial, but is alleviated when residing precisely on the blind spot.

Figure 8 also contains a line corresponding to bino-Higgsino DM in the MSSM. In this case, $y=g^{\prime} / \sqrt{2}$, and $\theta=\beta$ is restricted to be in the range $-\pi / 4$ to $+\pi / 4$ required for a perturbative top Yukawa coupling. Here, negative values of $\beta$ correspond to a negative $\mu$ parameter in the MSSM.

Lastly, figure 9 shows the theoretically available parameter space with $\Omega_{\chi}=\Omega_{\mathrm{DM}}$ in terms of more physical quantities: the DM mass, $M_{\chi}$, and the DM-Higgs coupling, $\partial M_{\chi} / \partial v=$ $c_{h \chi \chi}$. By definition, the blind spot is defined at the bottom of the plot where $\left|c_{h \chi \chi}\right|=0$. We can substitute the model inputs $M_{S}$ and $y$ for these more physical parameters using the relations

$$
\begin{aligned}
M_{S} & =M_{\chi}-\frac{1}{2} c_{h \chi \chi} v \frac{\Delta M^{2} B}{\Delta M^{2} B+c_{h \chi \chi} v\left(\Delta M^{2}+2 M_{\chi} B\right) / 2} \\
y^{2} & =-\frac{c_{h \chi \chi}}{v B^{2}}\left(\Delta M^{2}\left(M_{S}-M_{\chi}\right)+B\left(\Delta M^{2}+2 M_{\chi}\left(M_{S}-M_{\chi}\right)\right)\right) \\
\Delta M^{2} & =M_{D}^{2}-M_{\chi}^{2} \\
B & =M_{\chi}+M_{D} \sin 2 \theta .
\end{aligned}
$$

We have marginalized over all values of $\tan \theta$ positive (left) and negative (right) to fix $\Omega_{\chi}=$ 
$\Omega_{\mathrm{DM}}$ for various values of $M_{\chi^{ \pm}}=M_{D}$. In keeping with comparison to direct detection the sign of $c_{h \chi \chi}$ is also left undetermined. This issue will be discussed further in section 3.3.

For $\tan \theta>0$ both the upper and lower edges of the allowed range increase with $M_{\chi}$. The position of the upper edge depends strongly on $M_{D}$, and increases more quickly for small $M_{D}$, while the position of the lower edge is almost independent of $M_{D}$. This behavior is modified as $M_{\chi}$ and $M_{D}$ become degenerate, with the upper edge peaking at $M_{\chi} \approx M_{D}-100 \mathrm{GeV}$ and dropping steadily thereafter. The lower edge also drops below the general trend line in the same mass range. Both effects are due to coannihilation, which requires a spectrum with relatively suppressed Higgs couplings. The presence of a general lower bound on $c_{h \chi \chi}$ is due to the lack of a blind spot for $\tan \theta>0$. However, a small region with $c_{h \chi \chi} \rightarrow 0$ exists for $\tan \theta>0$ in the $M_{\chi} \rightarrow M_{D}$ limit as small cross-terms are required for mixing. Note that the absence of this region in figure 9 (a) is due to a binning artifact.

For $\tan \theta<0$, the behavior of the upper edge is similar to that for $\tan \theta>0$, though found at somewhat different values of $\left|c_{h \chi \chi}\right|$. There is no lower bound, however, due to the possibility of blind spot cancellations for $\tan \theta<0$. In terms of constraints, LUX currently excludes most of the $\tan \theta>0$ regions, including everything above the generic lower limit, and excludes a large portion of the $\tan \theta<0$ regions as well. XENON1T will further exclude most of the parameter space of this simplified model, though for the cases of extreme coannihilation and blind spots for $\tan \theta<0$, XENON1T will not be able to eliminate the model.

\subsection{Exclusion plots (thermal relic and marginal exclusion)}

So far, we have reduced the dimensionality of the parameter space by imposing $\Omega_{\chi}=\Omega_{\mathrm{DM}}$. However, we can further reduce the parameter space by fixing $\sigma^{\mathrm{SI}}$, which is controlled by $c_{h \chi \chi}$ coupling. In particular, we will now focus on the parameter space of thermal relic DM that exactly saturates present LUX limits or projected XENON1T reach (in addition to fixing $\Omega_{\chi}=\Omega_{\mathrm{DM}}$ ). This defines a space of marginally excluded, thermal relic DM models which can accommodate the observed abundance today. This space of marginally excluded thermal relics represents the class of models at the edge of direct detection limits. Thus, comparing the space fixed to XENON1T projected constraints against that fixed to current LUX limits unambiguously demonstrates the effect of improved direct detection sensitivity. This is aided by the additional benefit of reducing the parameter space to only two dimensions; hence the entire surviving parameter space can be described in a single plane. Moreover, because the direct detection limits bound $\sigma^{\mathrm{SI}}$ from above, the values $y$ shown can be identified as the maximal DM-Higgs coupling allowed by a given experiment. The corresponding $M_{\chi}$ should be interpreted as the minimal DM mass allowed by direct detection, at least for $M_{\chi}>200 \mathrm{GeV}$, when experimental limits on the cross-section begin to scale linearly as $\propto M_{\chi}$.

First we consider the parameter space of marginally excluded thermal relics in the $\left(M_{D}, M_{\chi}\right)$ plane for $\tan \theta<0$. For every pair of $\left(M_{\chi}, M_{D}\right)$, there are a maximum of four viable solutions - each with $c_{h \chi \chi}$ either positive or negative and $\tan \theta$ either positive or negative. From eq. (2.12),

$$
c_{h \chi \chi} \propto-\left(M_{\chi}+M_{D} \sin 2 \theta\right),
$$

so it is negative definite for $\tan \theta$ positive, leaving a maximum of three viable solutions. Furthermore, from the discussion in section 3.2 it is clear that the viable region for $\tan \theta>0$ occurs only for small values of $y$ and significant well-tempering, and thus would occupy only a small sliver along the $M_{\chi}=M_{D}$ line in this plane; an explicit scan confirms this assumption.

The two remaining interesting cases are shown in figure 10 for $\tan \theta<0$ and $c_{h \chi \chi}>0$ (left) or $c_{h \chi \chi}<0$ (right). For each point the required value of $y$ is shown, along with regions 


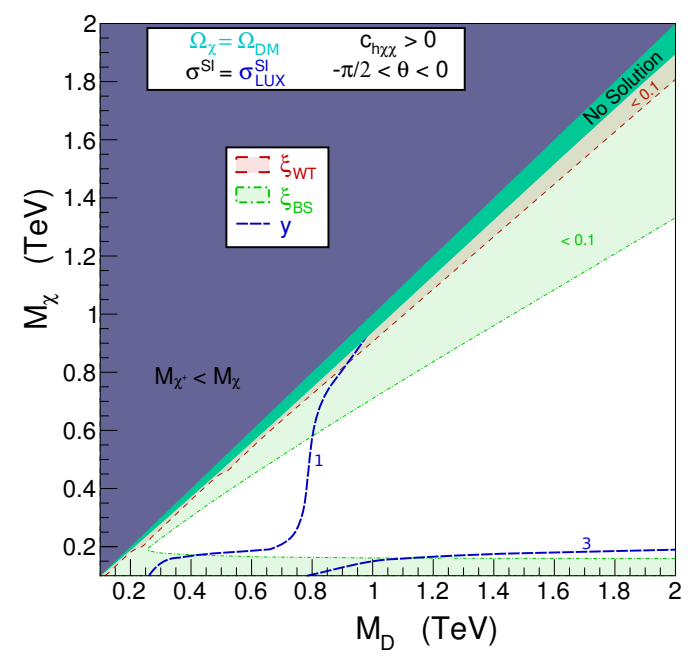

(a)

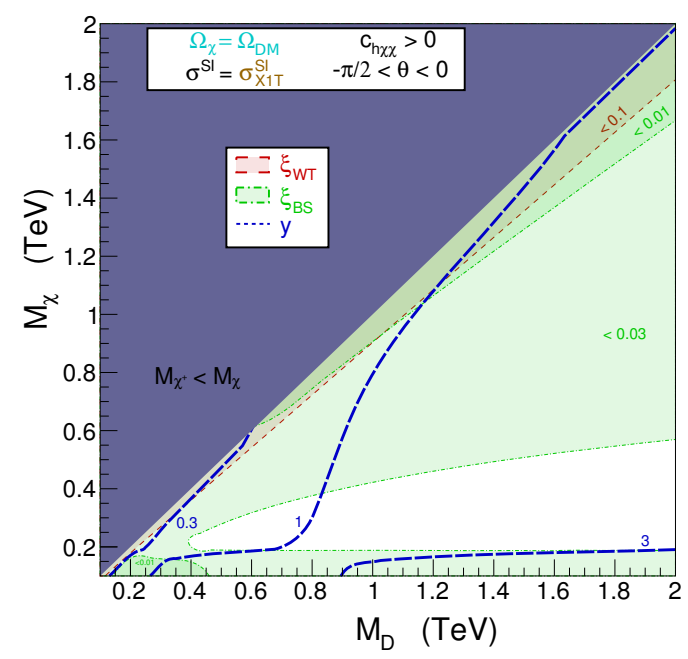

(c)

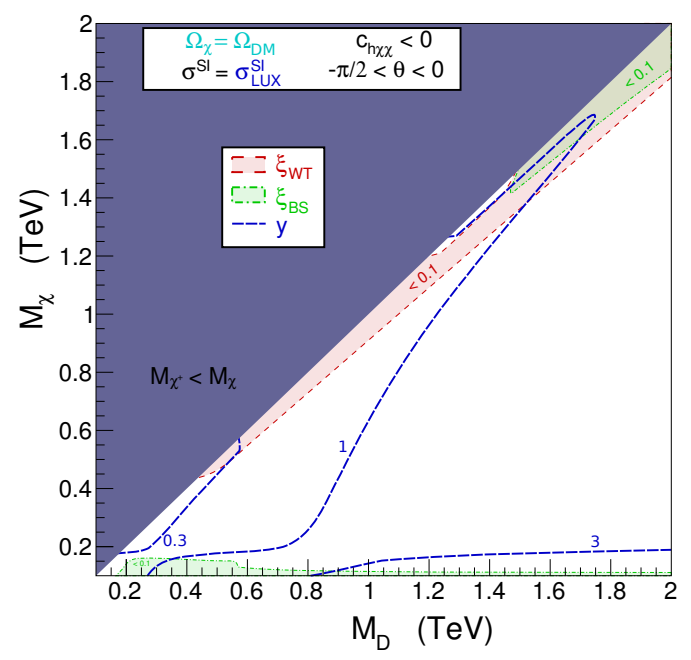

(b)

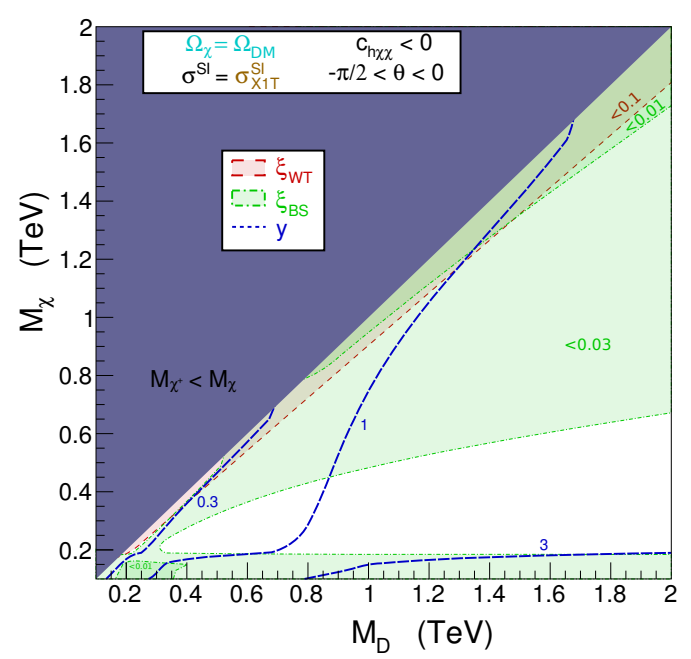

(d)

Figure 10. Tuning for models with fixed $\Omega_{\chi}$ and $\sigma^{S I}$ as a function of $M_{D}$ and $M_{\chi}$. Shown are points which satisfy the relic density constraint and exactly saturate $\sigma^{\mathrm{SI}}$ limits from LUX (top) or projected reach at XENON1T (bottom). Regions with significant tuning are shown for $\xi_{\mathrm{WT}}$ (red shaded) and $\xi_{\mathrm{BS}}$ (green shaded). The white regions correspond to parameters with lesser tuning than the shaded regions: $\xi_{\mathrm{WT}}, \xi_{\mathrm{BS}} \gtrsim 0.1$ for the upper panels, and $\xi_{\mathrm{WT}} \gtrsim 0.1$ and $0.1 \gtrsim \xi_{\mathrm{BS}} \gtrsim 0.03$ in the lower panels. The left panels correspond to $c_{h \chi \chi}>0$ and the right panels to $c_{h \chi \chi}<0$; in both cases $\tan \theta<0$.

showing various degrees of well-tempering and blind spot tuning. Models marginally excluded by LUX (top) have minimal tuning, with most regions subject to $\xi_{\mathrm{WT}}, \xi_{\mathrm{BS}} \gtrsim 0.1$ (shown in white) for both positive and negative $c_{h \chi \chi}$. For models within the marginal sensitivity of XENON1T, however, $\xi_{\mathrm{BS}}<0.1$ throughout the entire plane for both signs of $c_{h \chi \chi}$, and $\xi_{\mathrm{BS}}<0.03$ throughout most of the plane. While the parameter space remains viable for $\mathrm{DM}$, it is clear that some degree of fine-tuning is necessary for consistency with XENON1T projected limits. 


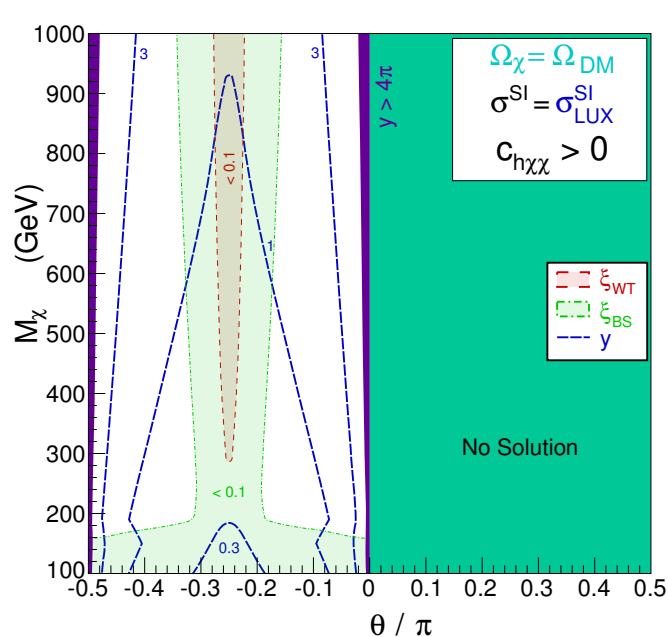

(a)

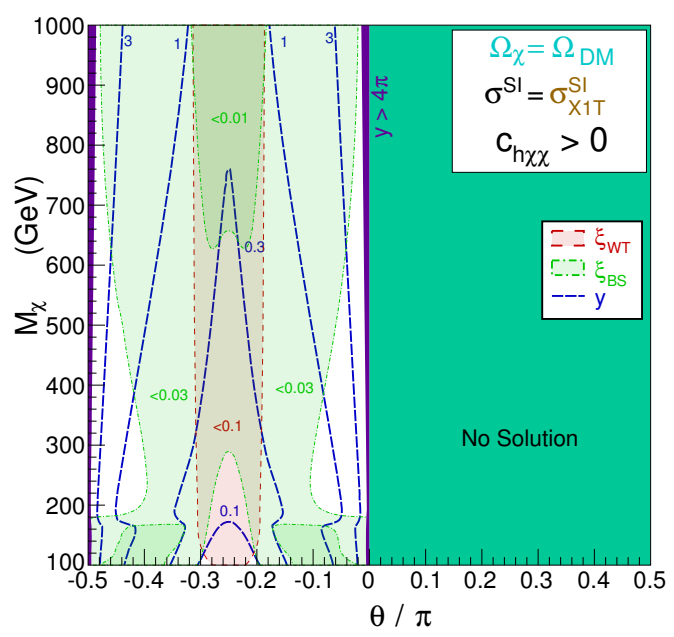

(c)

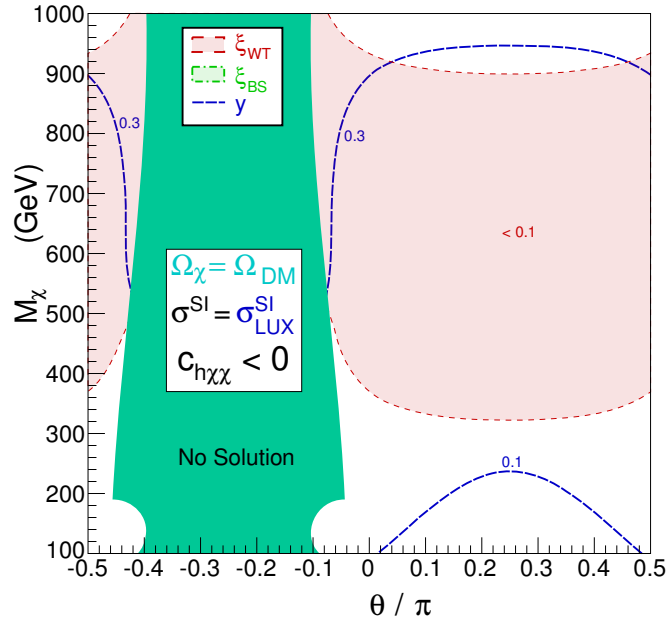

(b)

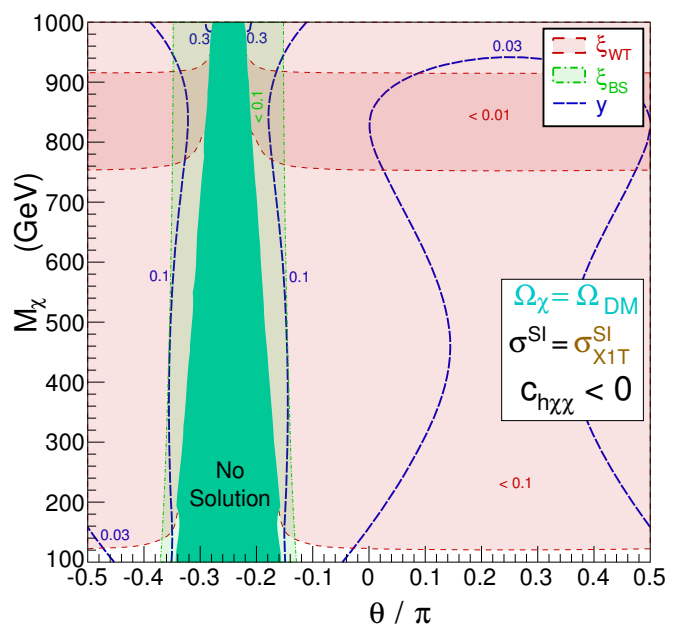

(d)

Figure 11. Tuning for models with fixed $\Omega_{\chi}$ and $\sigma^{S I}$ as a function of $\theta$ and $M_{\chi}$. Contours and regions shown are the same as in figure 10.

Figure 11 show the parameter space of marginally excluded thermal relic DM in the $\left(M_{\chi}, \theta\right)$ plane, with $M_{D}$ set to fix $\Omega_{\chi}=\Omega_{\mathrm{DM}}$. As discussed above, no solution exists for $c_{h \chi \chi}>0$ and $\tan \theta>0$. In principle, solutions exist throughout the plane for both signs of $c_{h \chi \chi}$ and $\tan \theta<0$. However, at sufficiently large values of $y$, the potential solutions for $c_{h \chi \chi}<0$ do not exist due to over-efficient annihilation, resulting in no solution near $\theta=-\pi / 4$. For $c_{h \chi \chi}>0$ the results mirror those shown in figure $10-$ there are significant regions with $\xi_{\mathrm{WT}}, \xi_{\mathrm{BS}} \gtrsim 0.1$ for LUX, while $\xi_{\mathrm{BS}}<0.1$ everywhere and $\xi_{\mathrm{BS}}<0.03$ throughout most of the region for XENON1T. In contrast, for $c_{h \chi \chi}<0$ the dominant fine-tuning lies in well-tempering. For $\tan \theta>0$, we find that $\xi_{\mathrm{BS}}=1$ by definition because no cancellations are possible to eliminate the DM-Higgs coupling. However, to evade direct detection, we still need tiny values of $y$, requiring coannihilation to produce $\Omega_{\chi}=\Omega_{\mathrm{DM}}$. This produces $\xi_{\mathrm{WT}}<0.1$ for $400 \mathrm{GeV} \lesssim M_{\chi} \lesssim 900 \mathrm{GeV}$ for LUX, while for XENON1T, $\xi_{\mathrm{WT}}<0.1$ throughout nearly the entire region shown and $\xi_{\mathrm{WT}}<0.01$ for $750 \mathrm{GeV} \lesssim M_{\chi} \lesssim 900 \mathrm{GeV}$. 


\section{Model B: singlet-doublet scalar DM}

Next, we will analyze the case of singlet-doublet scalar DM. This simplified model has qualitative similarities to the fermionic version discussed in section 3. However, there is an important physical difference: while a pure fermion singlet is inert, a pure scalar singlet can have renormalizable interactions to the SM through the Higgs boson. In particular, for scalar DM the quartic couplings between the DM and the Higgs can have a substantial effect on the thermal relic abundance and direct detection constraints. The addition of a quartic Higgs coupling to the singlet produces a line in the $M_{\chi}$ vs. $\sigma^{\text {SI }}$ plane consistent with the thermal relic density for pure singlet [61] DM, while a quartic Higgs coupling to the doublet shift the value of $M_{D}$ which gives $\Omega_{\chi}=\Omega_{\mathrm{DM}}$ independent of $M_{S}$ for pure doublet [71] DM. However, we are specifically interested in the case of mixed DM, whereby the dominant annihilation channels relevant to freeze-out derive from mixing of the singlet and non-singlet states. Thus, we will focus on the parameter space where the cubic term $A\left[S H D^{*}+\right.$ h.c. $]$ is large enough to induce significant mixing.

From the practical standpoint of analyzing constraints, scalar DM models tend to have more parameters than fermionic DM - in the case of singlet-doublet DM, the scalar model has seven while the fermionic has only four. The large number of parameters renders a comprehensive parameter scan like that used for fermions in section 3 impractical. Fortunately, four of the free parameters for scalar DM are quartic couplings of the Higgs directly to the singlet or doublet states, and thus do not induce singlet-doublet mixing. We will therefore focus on the case of $\lambda_{S}=\lambda_{D}=\lambda_{D}^{\prime}=\lambda_{D}^{\prime \prime}=0$ as the "minimal case" for mixed singlet-doublet scalar DM. As we move away from this simplifying limit, mixing becomes less important for the relic abundance and direct detection constraints, and the DM properties approach that of a pure singlet or doublet.

In principle, there are three distinct Higgs couplings to the doublet component of DM. However, only one combination of these enters into the couplings of the neutral mixed doublet state. Thus, many combinations of the three couplings will result in the same dynamics for the DM state alone. On the other hand, these different combinations will result in modified dynamics for processes involving DM and another doublet state, which here is limited to coannihilation for nearly pure doublet states. While these effects may be important in certain regions of parameter space, they are not the primary focus here.

The dominant effect of including Higgs couplings to pure states is a modification of $a_{h \chi \chi}$ and the coupling associated with the $\chi \chi h h$ operator. If re-expressed in terms of the singlet-doublet mixing angles, $a_{h \chi \chi}$ becomes [53]

$$
a_{h \chi \chi}=v\left(\lambda_{S} N_{S}^{2}+\left[\lambda_{D}+\lambda_{D}^{\prime}+\lambda_{D}^{\prime \prime}\right] N_{D}^{2}\right)-2 A N_{S} N_{D} .
$$

As shown in figure 3 , for the majority of the parameter space either $N_{S}^{2} \ll 1$ or $N_{D}^{2} \ll 1$, so either the singlet or doublet Higgs coupling contribution to $a_{h \chi \chi}$ and the associated $\chi \chi h h$ operator will be sub-dominant. Using this feature, we further simplify the parameter space by taking

$$
\begin{aligned}
\lambda_{S} & =\lambda_{D}=\lambda \\
\lambda_{D}^{\prime} & =\lambda_{D}^{\prime \prime}=0
\end{aligned}
$$

This simplified parameter space carries most of the qualitative features of the full theory, diverging primarily for $M_{S} \approx M_{D}$. 


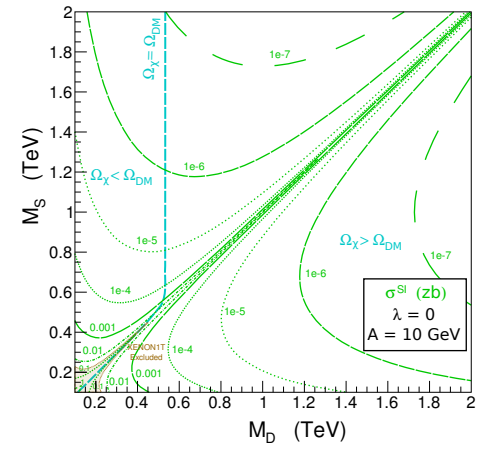

(a)

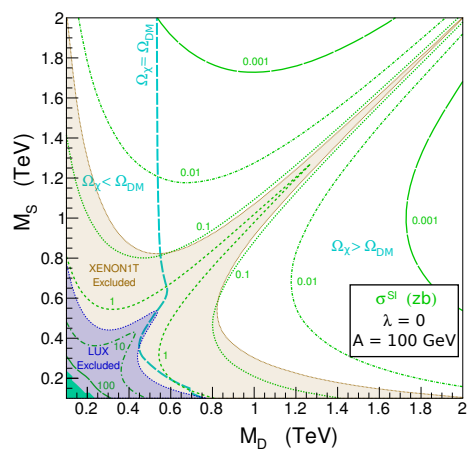

(b)

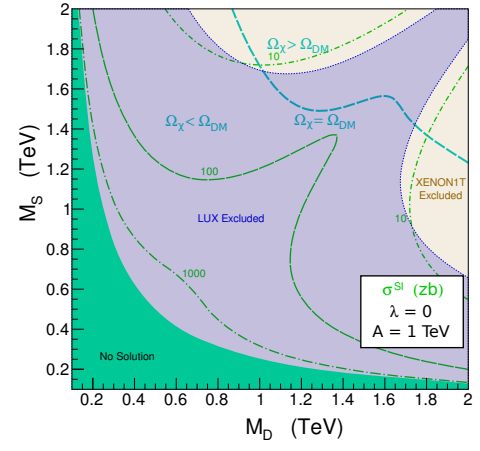

(c)

Figure 12. Direct detection prospects for $\lambda=0$ and $A=10,100,1000$ GeV. Contours shown are the same as in figure 5 .

\subsection{Exclusion plots (general)}

To begin, we analyze the unconstrained parameter space of scalar singlet-doublet DM. In figure 12 we restrict to the case $\lambda=0$, fixing $A=10,100,1000 \mathrm{GeV}$ from left to right. For $A=10 \mathrm{GeV}$, the associated cubic coupling is too weak to produce significant splitting or contribute to the relic density directly, thus requiring large mixing for $M_{S} \lesssim 500-$ $550 \mathrm{GeV}$. For $M_{S} \gtrsim 600 \mathrm{GeV}, \mathrm{DM}$ with $\Omega_{\chi}=\Omega_{\mathrm{DM}}$ becomes a nearly pure doublet with $M_{D} \approx 550 \mathrm{GeV}[21,71]$. In this case direct detection prospects are minimal, with XENON1T sensitivity only for $M_{S, D} \lesssim 200 \mathrm{GeV}$.

For $A=100 \mathrm{GeV}$, the position of the $\Omega_{\chi}=\Omega_{\mathrm{DM}}$ line changes substantially for $M_{S} \lesssim$ $800 \mathrm{GeV}$, shifting to larger values of $M_{D}$ as large as $\approx 750 \mathrm{GeV}$. However, it eventually asymptotes to pure doublet behavior for larger $M_{S}$. This shift is qualitatively similar to the enhanced Higgs-mediated annihilation for fermions in the case of large couplings, but the quantitative results diverge substantially. As discussed in section 3, while Majorana fermion DM can annihilate through the Higgs, this process appears at p-wave and only becomes important for large couplings. For scalars, however, annihilation via the Higgs is s-wave and interferes strongly with t-channel annihilation diagrams involving the other charged and neutral scalars. Qualitatively, the relative strength of Higgs annihilation can be seen in the direct detection coverage - the sensitivity of LUX and XENON1T shown in figure 12(b) is much weaker for equivalent $M_{\chi}$ than the full-plane coverage present for fermions in figures $5(\mathrm{a})$ and $5(\mathrm{c})$, but despite this achieving $\Omega_{\chi}=\Omega_{\mathrm{DM}}$ requires less welltempering, at least for $M_{S} \lesssim 800 \mathrm{GeV}$.

The relevance of annihilation through the Higgs is even more pronounced for $A=1 \mathrm{TeV}$. In this case, $\Omega_{\chi}=\Omega_{\mathrm{DM}}$ cannot be achieved for small DM masses due to Higgs-mediated annihilation. The $\Omega_{\chi}=\Omega_{\mathrm{DM}}$ line occurs at $M_{S} \gtrsim 1.2 \mathrm{TeV}$, corresponding to DM masses well above the value of $\sim 550 \mathrm{GeV}$ required for a pure doublet thermal relic. The $\Omega_{\chi}=\Omega_{\mathrm{DM}}$ line occurs at somewhat smaller DM mass for $M_{D}<M_{S}$ than for $M_{D}>M_{S}$ due to the more efficient annihilation from the typical doublet annihilation processes, but in both cases the relatively small mixing angle still produces large direct detection cross-sections. As a result, a portion of the $\Omega_{\chi}=\Omega_{\mathrm{DM}}$ line remains outside of LUX bounds despite the relatively large values of $\sigma^{\mathrm{SI}}$, though it can be probed at XENON1T.

The situation changes drastically if there are quartic couplings between the Higgs and the singlet and doublet components of the DM, shown in figure 13 for $\lambda= \pm 0.25$. The 
most distinctive feature is the possibility of $\Omega_{\chi}=\Omega_{\mathrm{DM}}$ for pure singlet DM, occurring at $M_{S} \approx 800 \mathrm{GeV}$ for a small mixing term $A=10 \mathrm{GeV}$. Furthermore, a Higgs coupling to the doublet also shifts the mass of the $\Omega_{\chi}=\Omega_{\mathrm{DM}}$ for pure doublet DM to $M_{D} \approx 650 \mathrm{GeV}$. For $A \ll \lambda v$ the Higgs couplings to pure states dominates, and the sign of $\lambda$ is irrelevant except for $M_{S} \approx M_{D}$. Direct detection sensitivity is strong even for the modest value of $\lambda= \pm 0.25$, with LUX sensitivity reaching $M_{S}$ or $M_{D}$ of just under $400 \mathrm{GeV}$, and XENON1T sensitivity reaching out to $M_{S}, M_{D} \lesssim 1.5 \mathrm{TeV}$, covering the entire $\Omega_{\chi}=\Omega_{\mathrm{DM}}$ line.

The sign of $\lambda$ becomes important for $A \sim \lambda v$. As can be seen from eq. (2.21), the contribution of $A \neq 0$ to the DM-Higgs coupling is always negative, leading to an enhancement of the couplings for $\lambda<0$ and a suppression for $\lambda>0$. The corresponding blind spot cancellation regions are located near but slightly offset above and below the $M_{S}=M_{D}$ line in figure 13(b) for $\lambda=0.25$, with no corresponding behavior present in figure 13(e) for $\lambda=-0.25$. For $\lambda=0.25, A=100 \mathrm{GeV}$, certain points in the blind spot region are actually consistent with $\Omega_{\chi}=\Omega_{\mathrm{DM}}$, while for $\lambda=-0.25, A=100 \mathrm{GeV}$ the entire $\Omega_{\chi}=\Omega_{\mathrm{DM}}$ contour is within the sensitivity range of XENON1T. A small region also exists for $\lambda=0.25, A=100 \mathrm{GeV}$ with $\Omega_{\chi}=\Omega_{\mathrm{DM}}$ for $M_{S}, M_{D} \lesssim 200 \mathrm{GeV}$, resulting from the cancellation in the Higgs coupling; by varying values of $A$ for appropriate suppression this low mass contour can be shifted anywhere with $M_{S}<M_{D}$.

For $A=1 \mathrm{TeV} \gg \lambda v$, the mixing term dominates for both signs of $\lambda$, so no blind spot is present; however, $\sigma^{\mathrm{SI}}$ is enhanced for $\lambda=-0.25$ relative to $\lambda=0.25$ throughout the plane and constraints are stronger, as shown in figures 13(c) and 13(f). As in figure 12(c), when $A=1 \mathrm{TeV}$, the Higgs coupling is so large that the majority of parameter space is already excluded by LUX. The relative sign of $\lambda$ also has a significant effect on the location of the $\Omega_{\chi}=\Omega_{\mathrm{DM}}$ line, with the enhanced coupling for $\lambda=-0.25$ pushing the contour to larger mass, while the suppressed coupling for $\lambda=0.25$ shifts the line to lower mass. As for $\lambda=0$, the portions of the $\Omega_{\chi}=\Omega_{\mathrm{DM}}$ lines in the region shown are not excluded by LUX, but are within XENON1T sensitivity for $\lambda= \pm 0.25$.

\subsection{Exclusion plots (thermal relic)}

Next, we consider the singlet-doublet scalar model for $\Omega_{\chi}=\Omega_{\mathrm{DM}}$. In keeping with our interest in mixed DM, we fix to various values of $\lambda$, leaving only three variables in the remaining parameter space, $\left(M_{S}, M_{D}, A\right)$. Within this sub-space, for fixed $\left(M_{S}, A\right)$ or $\left(M_{D}, A\right)$ a varying number of solutions exist for $\Omega_{\chi}=\Omega_{\mathrm{DM}}$, as evidenced by figures 12 and 13. In particular, for $\lambda \neq 0$ sufficiently large, a solution always exists for which $\Omega_{\chi}=\Omega_{\mathrm{DM}}$ is independent of $M_{D}$ and $M_{S}$, respectively, above a certain critical value. This limits the usefulness of an analog to figure 7 or figure 8 where either $M_{S}$ or $M_{D}$ is used to fix the relic density.

As such, we consider the $\left(M_{D}, M_{S}\right)$ plane in figure 14 with $\lambda=0, \pm 0.25$ and $A$ fixed to produce $\Omega_{\chi}=\Omega_{\mathrm{DM}}$. For $\lambda=0$, no solution is present for $M_{D}<M_{S}$ and $M_{D} \lesssim 550 \mathrm{GeV}$, as a nearly pure doublet is under-dense in this region. The same effect is present for $\lambda= \pm 0.25$ for $M_{D} \lesssim 650 \mathrm{GeV}$. Throughout the rest of the plane in figure 14(a), direct detection sensitivity to the $\Omega_{\chi}=\Omega_{\mathrm{DM}}$ scenario is strong, with the best sensitivity present for $M_{S} \approx M_{D}$ and dropping off as the masses become less degenerate. In fact, direct detection sensitivity grows at large DM mass because the mixing required for the thermal relic abundance requires $(v A)^{2} \sim M_{\chi}^{4}$ at large mass, resulting in $c_{h \chi \chi} \sim M_{\chi} / v$. The DM-nucleon cross-section thus scales as $\sigma^{\mathrm{SI}} \propto c_{h \chi \chi}^{2} \propto\left(M_{\chi} / v\right)^{2}$, which grows faster than the direct detection limits weaken: $\sigma_{\mathrm{LUX}}^{\mathrm{SI}}, \sigma_{\mathrm{X} 1 \mathrm{~T}}^{\mathrm{SI}} \propto M_{\chi}$. For $\lambda=0$, the blind spot is present for $M_{S}>M_{D}$ and $M_{D}$ near the "no solution" region. 


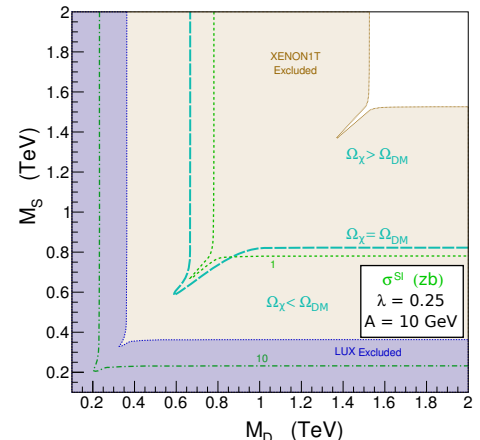

(a)

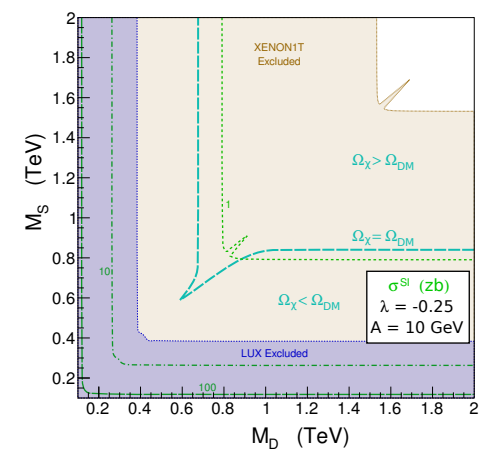

(d)

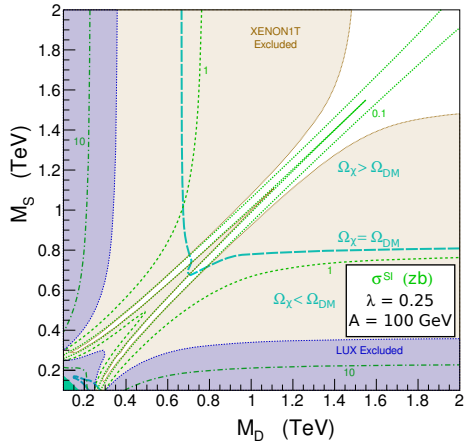

(b)

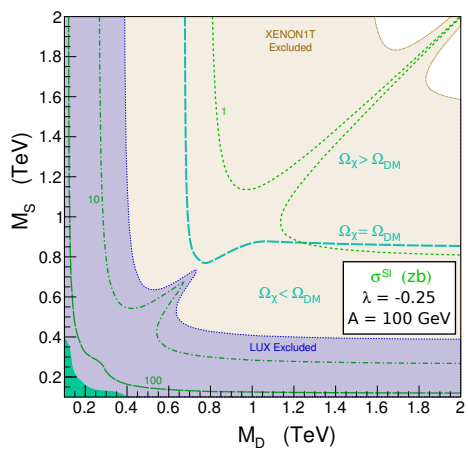

(e)

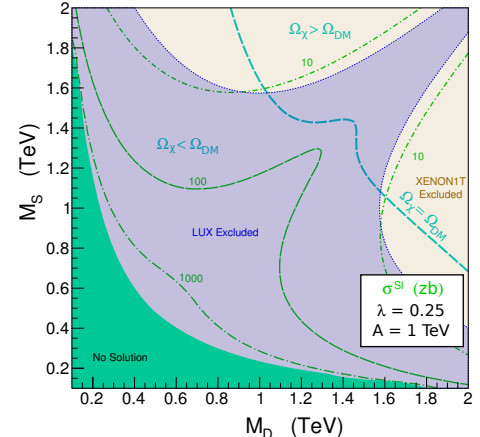

(c)

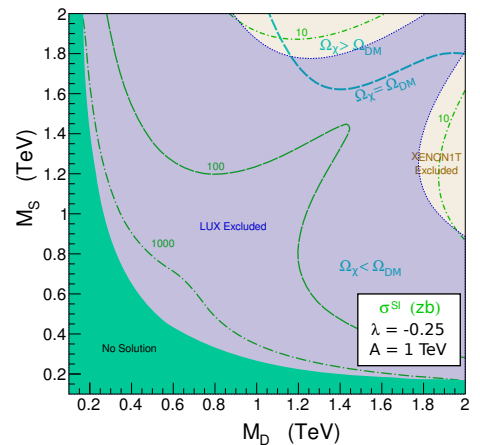

(f)

Figure 13. Direct detection prospects for $\lambda=0$ and $A=10,100,1000 \mathrm{GeV}$. Contours shown are the same as in figure 5 .

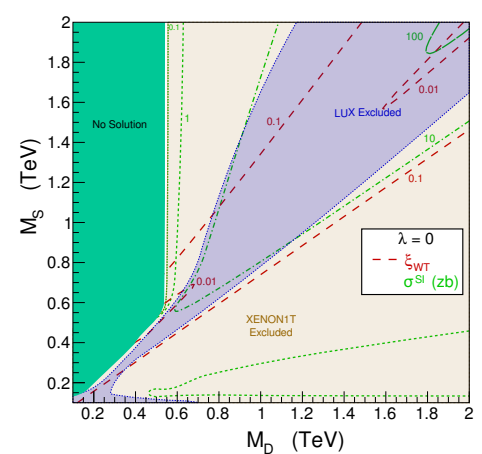

(a)

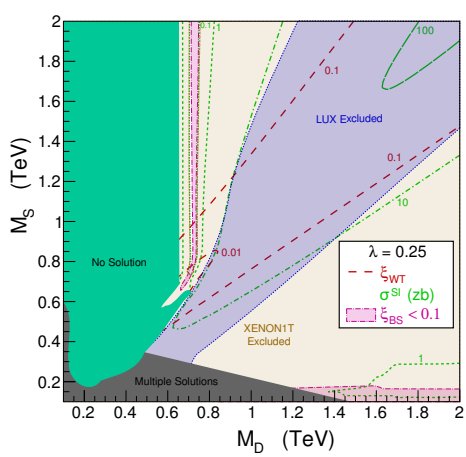

(b)

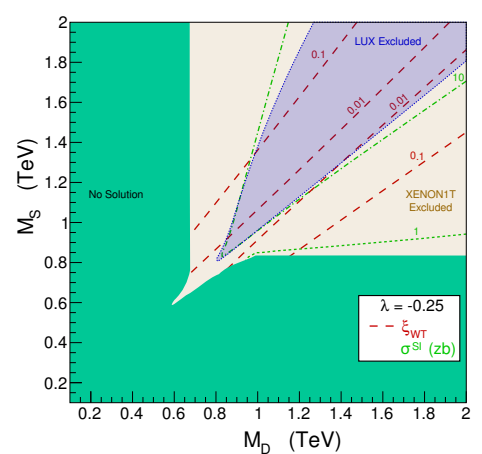

(c)

Figure 14. Well-tempering and direct detection prospects for fixed $\Omega_{\chi}=\Omega_{D M}$ as a function of $M_{D}$ and $M_{S}$. Contours shown are the same as in figure 7 , with the addition of a region corresponding to $\xi_{\mathrm{BS}}<0.1$ (pink shaded) in figure 14(b).

For $\lambda \neq 0$ the interplay of mixing and non-mixing Higgs couplings modifies the location of the "no solution" and blind spot regions. For $\lambda=-0.25$, no solution exists for $M_{S} \lesssim$ $800 \mathrm{GeV}$ except for $M_{S} \approx M_{D}$, as the Higgs-mediated annihilation cross-section is too large in this region. However, despite an enhanced Higgs coupling, the LUX exclusion is 


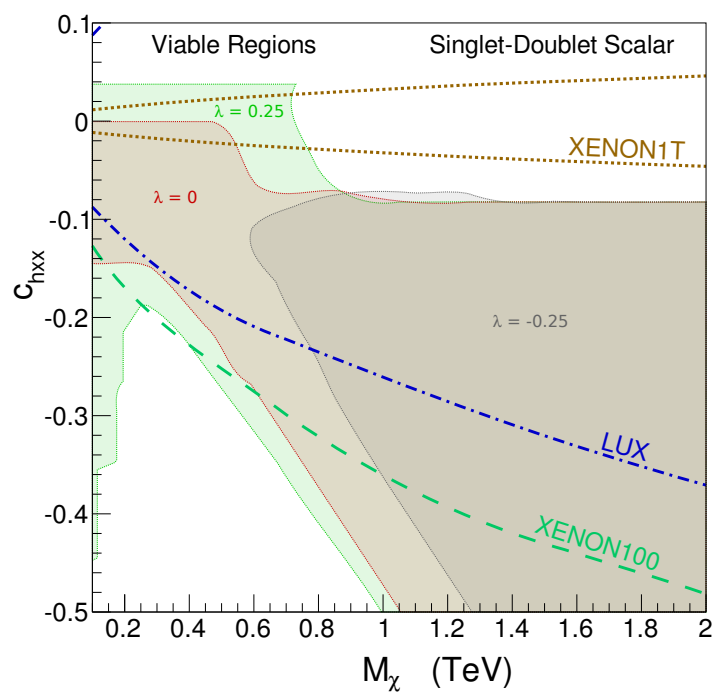

Figure 15. Viable regions with $\Omega_{\chi}=\Omega_{\mathrm{DM}}$ as a function of $M_{\chi}$ and $c_{h \chi \chi}$. Regions are shown for $\lambda=0$ (red shaded), $\lambda=0.25$ (green shaded) and $\lambda=-0.25$ (gray shaded) with $M_{D}$ fixed to produce the correct relic density. Also shown are upper limits on $\left|c_{h \chi \chi}\right|$ from XENON100 (teal dashed), LUX (blue dot-dashed), and projected upper limits from XENON1T (gold dashed).

marginally weaker for $\lambda=-0.25$ - the direct coupling alone is insufficient to saturate the LUX bound for $M_{S} \not \approx M_{D}$, but it does reduce the required degree of mixing necessary to produce $\Omega_{\chi}=\Omega_{\mathrm{DM}}$. However, the entire viable region for $\lambda=-0.25$ falls within project XENON1T sensitivity. For $\lambda=0.25$, the LUX exclusion is correspondingly stronger than for $\lambda=0$; however, the blind spot is shifted away from the "no solution" region to $M_{D} \approx 725 \mathrm{GeV}$ and $M_{S} \gtrsim 750 \mathrm{GeV}$. A region with multiple solutions for $\Omega_{\chi}=\Omega_{\mathrm{DM}}$ also exists for small $M_{S}$ and $\lambda=0.25$, resulting from the interplay of Higgs coupling cancellation with varying $A$. In this region XENON1T constrains most solutions, but a set exists for which the singlet and doublet states are highly mixed but $a_{h \chi \chi}$ vanishes due to a cancellation between the contributions, producing a blind spot.

The blind spot tuning measure, $\xi_{\mathrm{BS}}$, only has meaningful implications for the $\lambda>0$ parameter space. As defined in eq. (2.23), $\xi_{\mathrm{BS}}=1$ identically for $\lambda \leq 0$, with partial cancellation in the top term possible only for $\lambda>0$. This gives $\xi_{\mathrm{BS}} \lesssim 0.1$ throughout the region allowed by XENON1T in the $\lambda>0$ plane. The region has a widths of $50 \mathrm{GeV}$, which naïvely is somewhat thinner than $10 \%$ turning would indicate; however, the size of a finetuned region should be compared to the characteristic mass, making a $10 \%$ tuning reasonable for $M_{\chi} \approx 700 \mathrm{GeV}$.

The experimentally viable region for singlet-doublet scalar DM is depicted in figure 15 in the plane of physical variables, $\left(M_{\chi}, c_{h \chi \chi}\right)$. To produce this figure we have replaced the model parameters $\left(M_{S}, A\right)$ with $\left(M_{\chi}, c_{h \chi \chi}\right)$, and set $M_{D}$ to a value in order to accommodate $\Omega_{\chi}=\Omega_{\mathrm{DM}}$. As noted before, this produces multiple solutions at most points in each region, particularly for $\left|c_{h \chi \chi}\right| \gtrsim 0.1$. For $\left|c_{h \chi \chi}\right| \gtrsim 0.1$ we find that $\Omega_{\chi}<\Omega_{\mathrm{DM}}$ both for $M_{D} \approx M_{\chi}$ due to coannihilation and for $M_{D} \rightarrow \infty$ due to pure Higgs coupling. However, $\Omega_{\chi}>\Omega_{\mathrm{DM}}$ for an intermediate range of $M_{D}$ due to destructive interference between pure gauge and Higgs-mediated annihilation diagrams, producing at least two solutions for $\Omega_{\chi}=\Omega_{\mathrm{DM}}$.

According to eq. (2.21), $c_{h \chi \chi} \leq 0$ for $\lambda=0$. For $M_{\chi} \lesssim 550 \mathrm{GeV}$, arbitrarily small values of $c_{h \chi \chi}$ are viable, since a sufficiently small splitting $\left|M_{D}-M_{S}\right|$ can always be chosen 
to produce the correct degree of mixing through pure gauge diagrams. For $M_{\chi} \gtrsim 550 \mathrm{GeV}$, some degree of Higgs-mediated annihilation is required to produce $\Omega_{\chi}=\Omega_{\mathrm{DM}}$, and the region boundaries are set by the relative size of Higgs-mediated versus pure gauge annihilation diagrams. The upper boundary along $c_{h \chi \chi} \approx 0.8$ for $M_{\chi} \gtrsim 550 \mathrm{GeV}$ has the relic density set entirely by Higgs-mediated diagrams, with gauge diagrams suppressed by $M_{D} \gg M_{\chi}$. In this case $A^{2} / M_{D}^{2} \sim M_{\chi} / v$, so the underlying Higgs coupling must increase dramatically with $M_{\chi}$ and will eventually become non-perturbative. For larger values of $\left|c_{h \chi \chi}\right|$ the degree of mixing is increased, with the lower boundary set by near-maximal mixing. The largest values of $c_{h \chi \chi}$ are achieved when significant interference is present between pure gauge and Higgsmediated diagrams, resulting in $\mathrm{BR}\left(\chi \chi \rightarrow W^{+} W^{-}, Z Z\right) \rightarrow 0$ and $\mathrm{BR}(\chi \chi \rightarrow t \bar{t}) \rightarrow 1$. The nearly pure doublet case for $M_{\chi} \gtrsim 550 \mathrm{GeV}$ is found at intermediate points with $M_{S} \gg M_{\chi}$ and Higgs-mediated diagrams compensating for insufficient pure gauge annihilation.

For $\lambda \neq 0$, the viable region is modified significantly at low mass but remains similar in shape at high mass. This is a result of the scaling of the terms in eq. (2.21), with the unmixed coupling term $\lambda v$ becoming sub-dominant for increasing $M_{\chi}$, while the term proportional to $A^{2}$ remains constant or increases with increasing $M_{\chi}$. For $\lambda=0.25$, positive $c_{h \chi \chi}$ is possible for $M_{\chi} \lesssim 700 \mathrm{GeV}$, and significantly larger values of $\left|c_{h \chi \chi}\right|$ are viable for $M_{\chi} \lesssim 200 \mathrm{GeV}$ due to interference effects in annihilation to gauge bosons for masses insufficiently large to allow for annihilation to $t \bar{t}$. For large $M_{\chi}$, however, the upper boundary asymptotes to $c_{h \chi \chi} \approx 0.8$ as in the $\lambda=0$ case, while the lower boundary is offset to more negative $c_{h \chi \chi}$ by a small value. For $\lambda=-0.25$, no solution is present for $M_{\chi} \lesssim 600 \mathrm{GeV}$ - both contributions to $c_{h \chi \chi}$ are negative, and the resulting value always produces $\Omega_{\chi}<\Omega_{\mathrm{DM}}$. While relatively large values of $\left|c_{h \chi \chi}\right|$ were feasible at low mass for $\lambda=0.25$, in such cases $A$ and $\left|M_{D}-M_{S}\right|$ could be relatively large due to cancellation between the contributions, resulting in a suppression of coannihilation contributions, while for $\lambda=-0.25$ the contributions are always additive. For large $M_{\chi}$ the upper boundary of the $\lambda=-0.25$ region also asymptotes to $c_{h \chi \chi} \approx 0.8$, while the lower boundary is offset to less negative $c_{h \chi \chi}$ by an amount somewhat larger than the offset for $\lambda=0.25$.

As previously shown in figure 14, the constraints from direct detection experiments become stronger for larger masses in figure 15. As noted in section 2.2, direct detection limits place a constraint on the quantity $c_{h \chi \chi}^{2} / M_{\chi}$, while the region with a viable thermal relic abundance is bounded by constant $c_{h \chi \chi}$ and constant $c_{h \chi \chi} / M_{\chi}$. The blind spot for small $M_{\chi}$ remains constrained to low $M_{\chi}$, though larger values of $M_{\chi}$ would become viable for increased $\lambda$. A significant region of parameter space remains unconstrained by LUX limits, particularly at large mass, but XENON1T projected sensitivity covers all viable regions except for the low mass blind spot. If XENON1T yields null results, then theories with negative $\lambda$ will be strongly constrained.

\section{Model C: singlet-triplet scalar DM}

Finally, we examine the experimental limits on mixed singlet-triplet scalar DM. The phenomenology of the singlet-triplet scalar model is similar to that of the singlet-doublet scalar model - the relic density is set primarily by DM-gauge interactions and Higgs-mediated diagrams. Moreover, these contributions can interference substantially in a way that strongly affects the final relic density determination. However, several features significantly alter the detailed phenomenology. First, the cubic DM-gauge interaction vanishes because of the $\mathrm{SU}(2)_{L}$ symmetry. The leading DM-gauge interaction is quartic, and has a coupling which 
is effectively four times stronger than in the singlet-doublet scalar case simply due to group theory factors. Thus, the model can accommodate $\Omega_{\chi}=\Omega_{\mathrm{DM}}$ primarily through gauge interactions with sufficient well-tempering up to larger values of $M_{\chi}$. Secondly, for a given mixing angle, the trilinear DM-Higgs coupling is larger by a factor of 2 for singlet-triplet DM as compared to singlet-doublet DM. This modifies the relative strength of contributions to annihilation and scattering. More significantly, however, is the DM-Higgs quartic interaction induced by the $\kappa$ mixing term. While a similar interaction is induced at tree level by the mixing term in the singlet-doublet scalar case, the contribution from the direct coupling for the singlet-triplet is larger for a spectrum with similar mass splittings.

Singlet-triplet scalar DM involves the same basic processes as singlet-doublet scalar $\mathrm{DM}$, so in the following analysis we focus primarily on the differences between these theories. Once again either $N_{S}^{2} \ll 1$ or $N_{T}^{2} \ll 1$ for most of the parameter space, so the region where $\lambda_{S}$ and $\lambda_{T}$ both play an important role in the dynamics is limited to $M_{S} \approx M_{T}$; thus for simplicity we set

$$
\lambda_{S}=\lambda_{T}=\lambda
$$

In the singlet-triplet case this is a somewhat better approximation, as the absence of additional quartic couplings reduces the range of possible divergent results for $M_{T} \not \approx M_{S}$.

\subsection{Exclusion plots (general)}

To analyze the unconstrained singlet-triplet DM parameter space, we fix $\lambda=0$ to eliminate the effects of the Higgs-DM quartic couplings. In the case of singlet-triplet DM, mixing is controlled by the dimensionless coefficient $\kappa$, which we set to $\kappa=0.3,3$ in figure 16 . The top two panels of figure 16 depict the thermal relic abundance and direct detection sensitivity in the $\left(M_{T}, M_{S}\right)$ plane. For the case of $\kappa=0.3$ in figure $16(\mathrm{a}), M_{S}$ and $M_{T}$ must be very degenerate to accommodate a thermal relic consistent with observations, and $\sigma^{\mathrm{SI}}$ is small throughout the plane. For $M_{S} \not \approx M_{T}$, LUX only constrains $M_{S}, M_{T} \lesssim 200 \mathrm{GeV}$, and even XENON1T only has sensitivity for $M_{S}, M_{T} \lesssim 400 \mathrm{GeV}$. In figure 16(b), the relatively large value of $\kappa=3$ shifts the $\Omega_{\chi}=\Omega_{\mathrm{DM}}$ line substantially, primarily due to an increase in the annihilation process $\chi \chi \rightarrow h h$ with $\kappa$. This depletes the DM abundance and implies that very large DM masses - upwards of multi- $\mathrm{TeV}$ - may be necessary to accommodate the observed DM relic abundance. While the both LUX and XENON1T strongly constrain the plane, with generic sensitivity to $M_{S}, M_{T} \lesssim 800 \mathrm{GeV}$ and $M_{S}, M_{T} \lesssim 1.5 \mathrm{TeV}$ respectively, the shift in the $\Omega_{\chi}=\Omega_{\text {DM }}$ line places some regions with a viable thermal relic beyond XENON1T reach.

The lower panels of figure 16 show the same information as the top panels, only plotted in the $\left(M_{T}-M_{S}, M_{S}\right)$ plane so as to focus on the diagonal region in which the singlet and triplet are well mixed. Sensitivity at direct detection experiments to the $M_{S} \approx M_{T}$ region is significantly improved relative to other parts of the plane. For $\kappa=0.3$ LUX bounds remain weak, but the projected limits from XENON1T for $\kappa=0.3$ are covering the $\Omega_{\chi}=\Omega_{\mathrm{DM}}$ contour up to $M_{S} \lesssim 500 \mathrm{GeV}$ and bound $M_{S} \approx M_{T} \lesssim 900 \mathrm{GeV}$. While experimental sensitivity improves for $\kappa=3$, with reach for $M_{S} \approx M_{T}>3 \mathrm{TeV}$, even in this case the $\Omega_{\chi}=\Omega_{\mathrm{DM}}$ contour is only within XENON1T reach for $M_{S} \lesssim 1.5 \mathrm{TeV}$.

The cases of $\lambda= \pm 0.25$ are shown in figure 17, which is otherwise identical to the upper panels of figure 16. As can be seen from eq. (2.28), a blind spot cancellation can occur if $\lambda$ is positive, in which case there will be destructive interference against Higgs exchange arising from the $\kappa$ induced mixing. This blind spot is visible in figure 16(b) for points near (but not directly on) the $M_{S} \approx M_{T}$ line. Similarly to the behavior in figure 13(b) for 


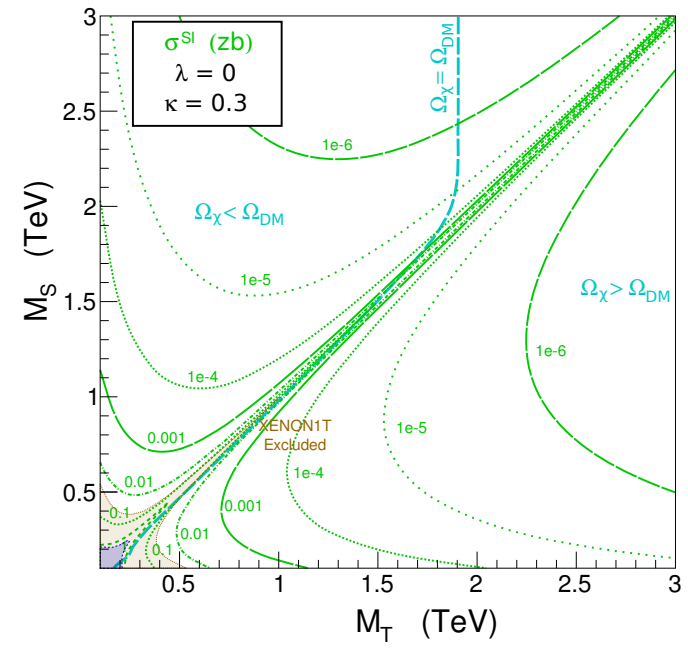

(a)

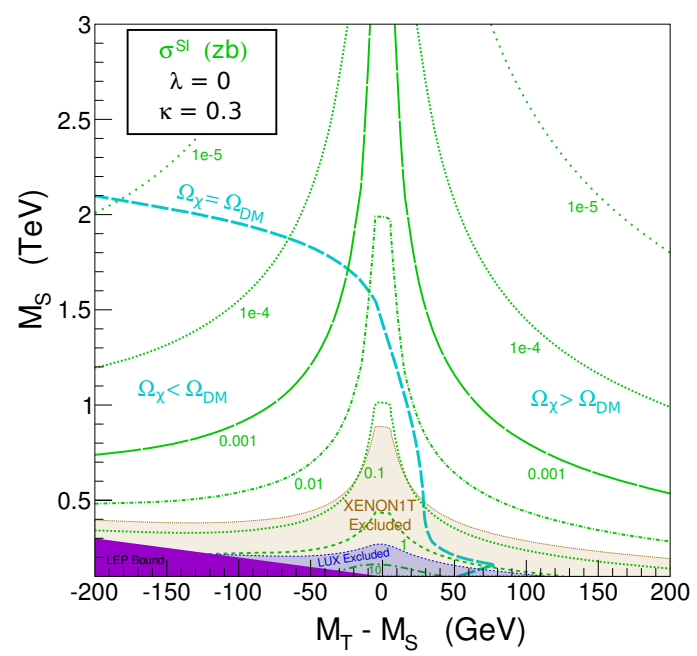

(c)

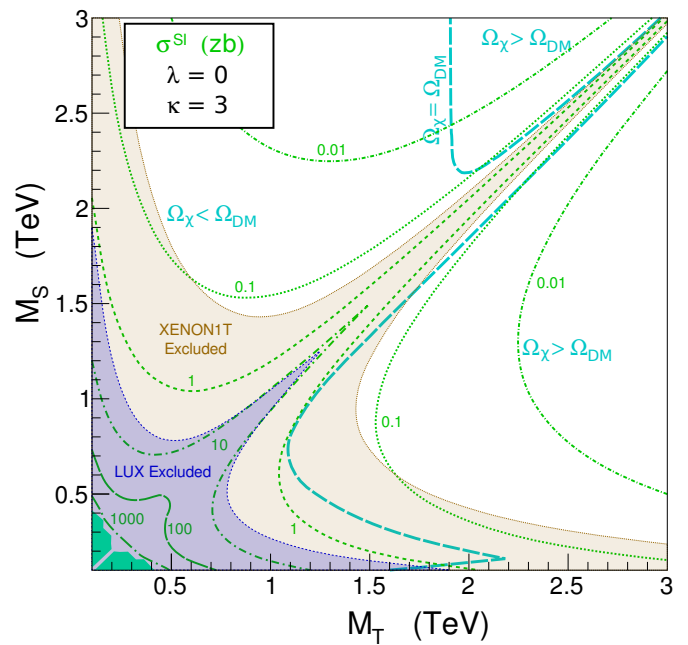

(b)

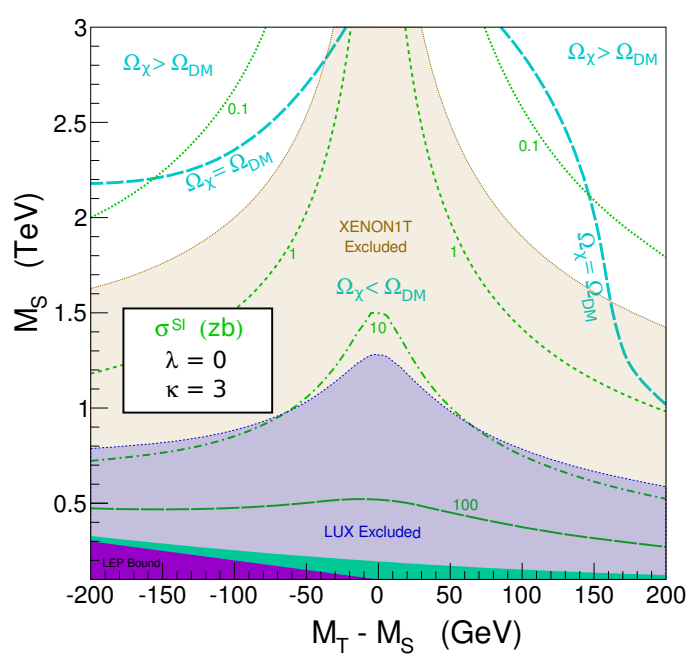

(d)

Figure 16. Direct detection prospects for $\lambda=0$ and $\kappa=0.3,3$. Contours shown are the same as in figure 5 .

singlet-doublet scalars, the DM-Higgs coupling is controlled by mixing induced by $\kappa$ near $M_{S} \approx M_{T}$. Away from this line, the DM-Higgs coupling is controlled by the direct quartic DM-Higgs couplings proportional to $\lambda$. Partial cancellation is also present in figure 16(a), though $\kappa=0.3$ is too small to produce a true blind spot for $\lambda=0.25$. For $\lambda=-0.25$ the direct detection cross-sections is increased relative to $\lambda=0$, with a corresponding LUX constraint of $M_{S}, M_{T} \gtrsim 1 \mathrm{TeV}$ for even moderate mixing. Meanwhile, XENON1T will exclude $M_{S}, M_{T} \lesssim 1.6 \mathrm{TeV}$, with even greater sensitivity for $M_{S} \approx M_{T}$.

\subsection{Exclusion plots (thermal relic)}

For the singlet-triplet scalar model, we set $\Omega_{\chi}=\Omega_{\text {DM }}$ by varying $\kappa$ in the $\left(M_{T}, M_{S}\right)$ plane in order to produce the observed relic density. Figure 18 depicts the viable parameter space of thermal relic singlet-triplet DM subject to current constraints from LUX and projected reach 


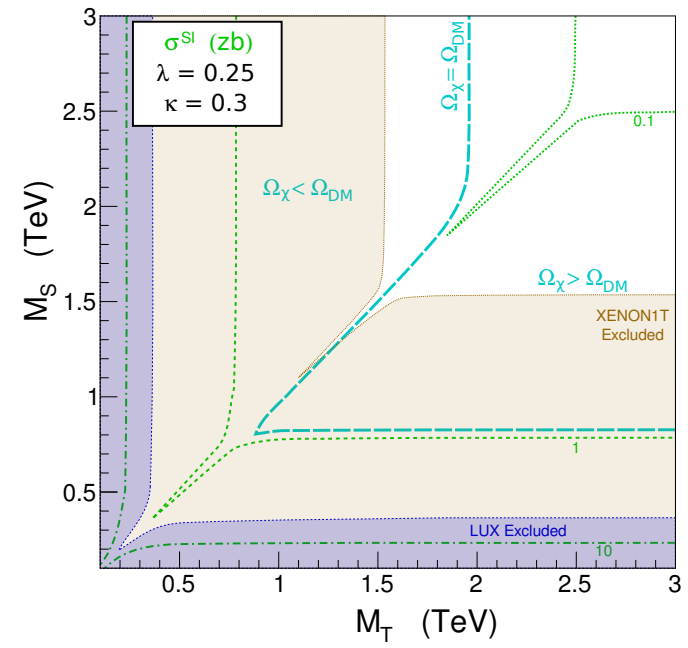

(a)

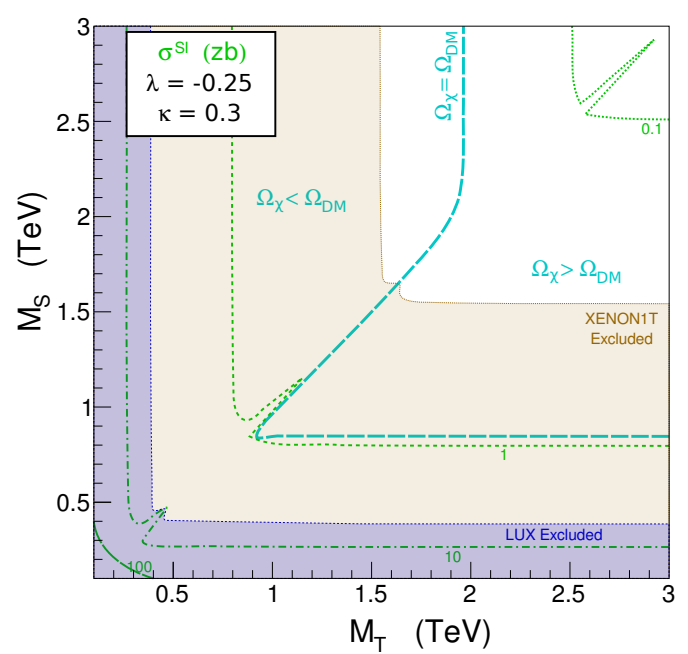

(c)

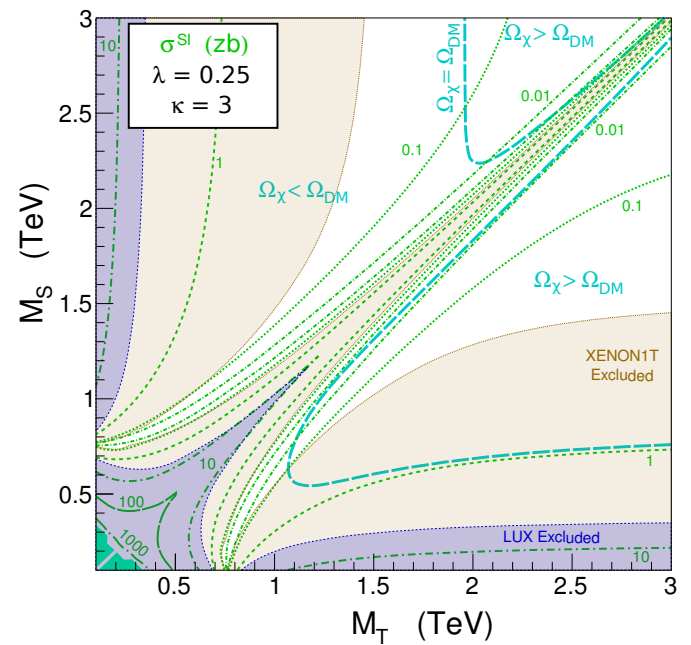

(b)

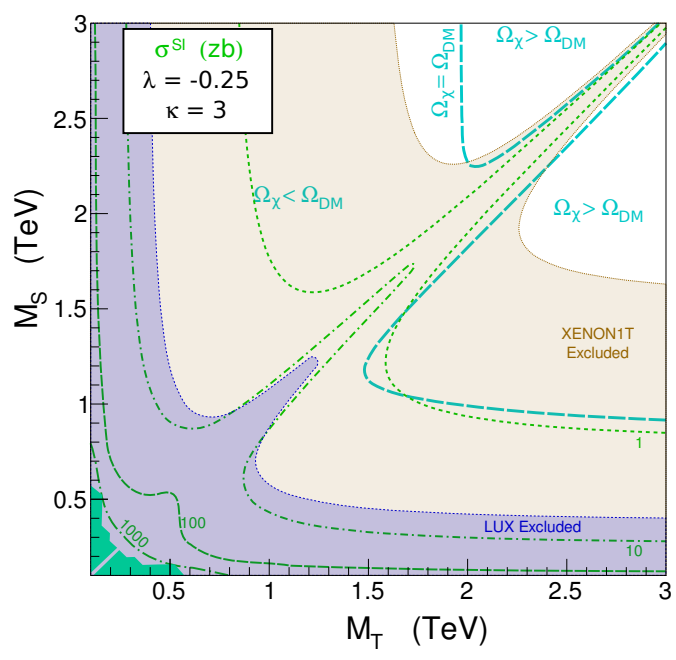

(d)

Figure 17. Direct detection prospects for $\lambda \neq 0$ and $\kappa=0.3,3$. Contours shown are the same as in figure 5 .

from XENON1T. For $\lambda=0$, there is no viable thermal relic for $M_{T}<M_{S}$ and $M_{T} \lesssim 1.9 \mathrm{TeV}$, since $\Omega_{\chi}<\Omega_{\mathrm{DM}}$ for all such models. For $M_{T}>M_{S}$ and $1.9 \mathrm{TeV} \lesssim M_{T}<M_{S}, \Omega_{\chi}=\Omega_{\mathrm{DM}}$ can be achieved throughout for sufficiently large $\kappa$, with $M_{T} \approx 1.9 \mathrm{TeV}, M_{S} \gg M_{S}$ corresponding to pure triplet DM with the correct thermal relic density $[21,71]$. Moreover, $\sigma^{\mathrm{SI}}$ varies by just over an order of magnitude over most of the viable range shown. This occurs because the dominant annihilation channel, $\chi \chi \rightarrow h h$, scales with mixing terms in the same way as the direct detection cross-section when $\kappa$ is sufficiently large. Hence, the direct detection and relic abundance are correlated. For $M_{S} \approx M_{T}$ annihilation is enhanced for identical values of $\kappa$ reducing $\sigma^{\mathrm{SI}}$ at large masses by roughly an order of magnitude. Because an equivalent value of $a_{h \chi \chi}$ implies stronger annihilation than in the singlet-doublet scalar case, the current LUX bounds only constrain a few points at small mass. However, because $\sigma^{\mathrm{SI}}$ is only weakly dependent on DM mass, XENON1T projected bounds constrain the majority of 


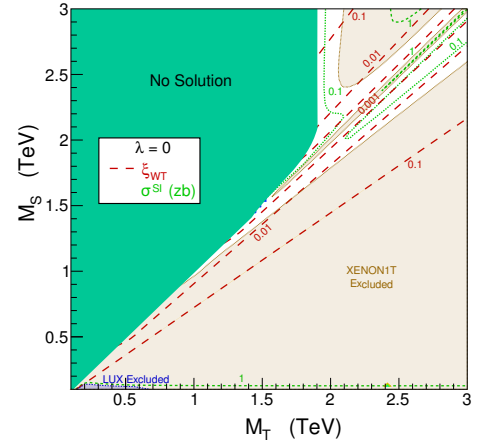

(a)

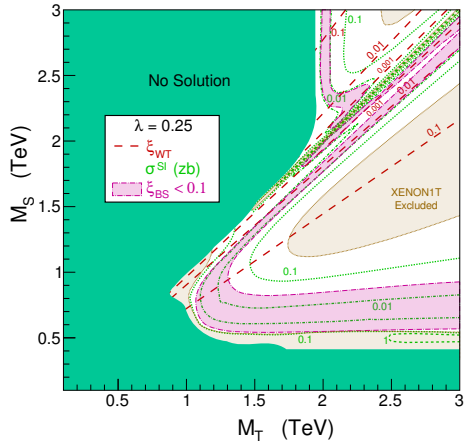

(b)

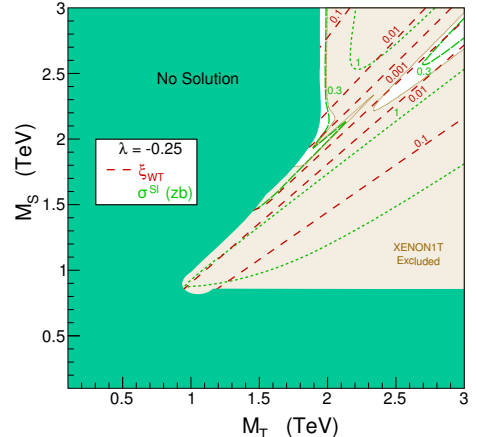

(c)

Figure 18. Well-tempering and direct detection prospects for fixed $\Omega_{\chi}=\Omega_{\mathrm{DM}}$ as a function of $M_{T}$ and $M_{S}$. Contours shown are the same as in figure 7 .

the parameter space shown. The only blind spot occurs along the edge of the "no solution" region for $M_{T} \approx 1.9 \mathrm{TeV}$, where the $\mathrm{DM}$ is nearly pure triplet.

Unlike for the singlet-doublet scalar case, for $\lambda=0.25$ in figure 18(b) the "no solution" region extends into the low mass $M_{S}<M_{T}$ region. For $M_{S} \lesssim 400 \mathrm{GeV}$, the direct singletHiggs quartic coupling results in $\Omega_{\chi}<\Omega_{\mathrm{DM}}$, and increasing $\kappa$ sufficiently to cancel the direct coupling contributions induces sufficient annihilation in other channels that $\chi$ is remains under-abundant, resulting in no viable solution for any value of $\kappa$. The allowed region still extends to lower $M_{S}$ than the $\Omega_{\chi}=\Omega_{\mathrm{DM}}$ contour in figure $17(\mathrm{a})$, however, and a blind spot occurs at $M_{S} \approx 650 \mathrm{GeV}$ consistent with this cancellation. This blind spot extends to large masses below and roughly parallel to the $M_{S}=M_{T}$ line. A second blind spot lies above this line and extends to large $M_{S}$ for $M_{T} \approx 2 \mathrm{TeV}$. The blind spot region is larger here in comparison to the singlet-doublet scalar case because the value of $a_{h \chi \chi}$ required to accommodate the observed relic density is significantly smaller. In the singlet-doublet case, gauge interactions are insufficient to set $\Omega_{\chi}=\Omega_{\mathrm{DM}}$ for any mixing angle for $M_{\chi} \gtrsim 550 \mathrm{GeV}$, and the induced DM-Higgs quartic coupling is relatively small. For singlet-triplet scalar DM, however, gauge interactions are strong throughout the entire range shown, and the DM-Higgs quartic coupling is sufficiently large that small values of $c_{h \chi \chi}$ are viable and even preferred. LUX has no constraining power for $\lambda=0.25$, and XENON1T sensitivity has only moderate coverage of the parameter space.

For $\lambda=-0.25$ in figure $18(\mathrm{c})$, the "no solution" region covers $M_{S} \lesssim 850 \mathrm{GeV}$ for $M_{S}<M_{T}$. No blind spot regions exist for $\lambda=-0.25$, but the relative strength of the gauge and four point interactions produces regions with small $\sigma^{\mathrm{SI}}$ along the upper portion of the "no solution" boundary and along the high mass $M_{S} \approx M_{T}$ line. LUX has no constraining power in the plane, and the regions with small $\sigma^{\mathrm{SI}}$ avoid even XENON1T reach despite the lack of true blind spot behavior. For both $\lambda=0$ and $\lambda=-0.25$ well-tempering of at least $\xi_{\mathrm{WT}} \lesssim 0.1$ is required to avoid XENON1T projected bounds except for nearly pure triple $\mathrm{DM}$, although most of the viable regions have well-tempering $\xi_{\mathrm{WT}} \lesssim 0.01$.

As in the singlet-doublet scalar case, for $\lambda \leq 0$ the blind spot fine-tuning is trivial, $\xi_{\mathrm{BS}}=1$, and $\xi_{\mathrm{BS}}$ is only physically meaningful when $\lambda>0$. For $\lambda>0$, however, the degree of fine-tuning needed to produce cancellation is significantly smaller than in the singlet-doublet scalar case. The pink region in figure 18(b) has tuning of $\xi_{\mathrm{BS}} \lesssim 0.1$ and covers approximately half of the area left viable after XENON1T. However, the remaining unshaded area avoids 


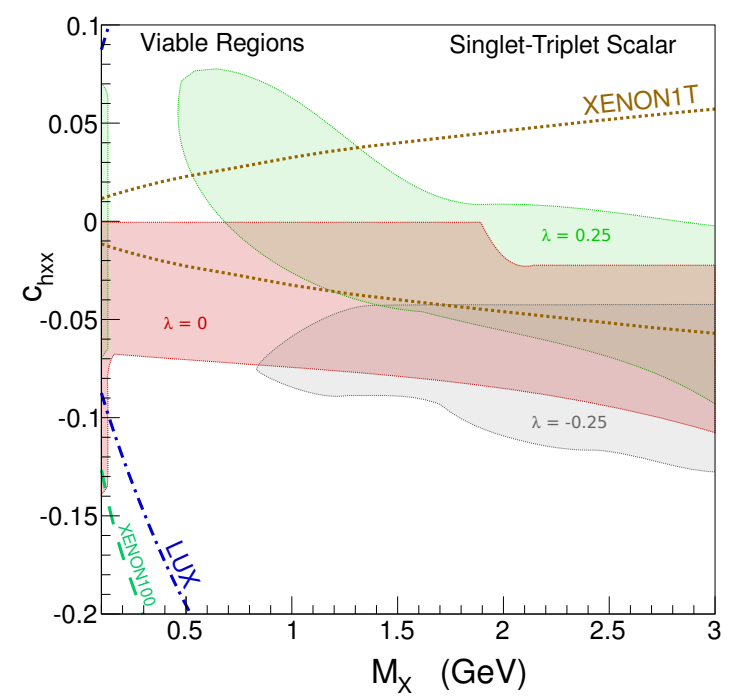

Figure 19. Viable regions with $\Omega_{\chi}=\Omega_{\mathrm{DM}}$ as a function of $M_{\chi}$ and $c_{h \chi \chi}$. Contours and regions shown are the same as in figure 15 .

XENON1T sensitivity with minimal tuning, $\xi_{\mathrm{BS}} \gtrsim 0.1$. The lower portion of this allowed area also has minimal tuning from well-tempering, $\xi_{\mathrm{WT}}>0.1$. Hence, for $\lambda>0$, singlet-triplet DM can accommodate viable thermal relic DM with minimal tuning.

The viable parameter space of singlet-triplet DM is depicted in the physical $\left(M_{\chi}, c_{h \chi \chi}\right)$ plane in figure 19. For $\lambda=0$, the behavior is similar to the singlet-doublet case, except the region is "stretched" horizontally and "squeezed" vertically $-c_{h \chi \chi}$ can be close to zero up to $M_{\chi} \lesssim 1.9 \mathrm{TeV}$, and the increase of $\left|c_{h \chi \chi}\right|$ along the lower boundary is more gradual. A small region extending to $c_{h \chi \chi} \approx-0.14$ is allowed for very low mass, where $M_{\chi}$ is below the Higgs production threshold and thus $\kappa$ can be significantly larger. For $\lambda=0.25$, most of the viable region is restricted to $M_{\chi} \gtrsim 500 \mathrm{GeV}$, while for $\lambda=-0.25, M_{\chi} \gtrsim 700 \mathrm{GeV}$ is required. For $M_{\chi} \gtrsim 500 \mathrm{GeV}$ the behavior for $\lambda= \pm 0.25$ is similar to the singlet-doublet scalar case. For all of the choices $\lambda=0, \pm 0.25$, there are parameter regions which are beyond the projected reach of XENON1T. In contrast to the singlet-doublet scalar case, the sensitivity of direct detection experiments weakens as $M_{\chi}$ increases.

\section{Conclusions and future directions}

Simplified models are a powerful tool for studying the generic behavior of WIMP DM. Theories in which DM couples to the SM via the Higgs are of particular interest because Higgsmediated DM-nucleon scattering is just now being probed by the current generation of direct detection experiments. In this paper we have constructed and analyzed simplified models of mixed DM describing a stable particle composed of a mixture of a singlet and an electroweak doublet or triplet. In these models DM undergoes thermal freeze-out through electroweak interactions to accommodate the observed DM relic abundance. Mixing between the singlet and non-singlet states is induced via DM-Higgs couplings, and is in general correlated with signals in direct detection.

We have determined the viable parameter space of these models subject to current LUX limits and the projected reach of XENON1T. Present experimental constraints from LUX place stringent limits on mixed DM models, with a DM mass of at least a few hundred GeV in 
most cases. The projected reach of XENON1T is significantly stronger, extending to masses of at least $1 \mathrm{TeV}$, and in many cases larger. Using simplified models of mixed DM, we have identified direct detection blind spots, which are parameter regions at which $\sigma^{\mathrm{SI}}$ vanishes identically, nullifying experimental limits on spin independent DM-nucleon scattering. Finally, we have quantified the degree of fine-tuning required for mixing angles $\left(\xi_{\mathrm{WT}}\right)$ and for blind spot cancellations $\left(\xi_{\mathrm{BS}}\right)$ required for thermal relic DM which is experimentally viable. Our results for each of our simplified models are summarized in the discussion below.

First, we studied singlet-doublet Majorana fermion DM, which is a generalization of mixed bino-Higgsino DM in the MSSM or singlino-Higgsino DM in the NMSSM. In these models the observed thermal relic density can be produced for $M_{\chi}<M_{D} \lesssim 1 \mathrm{TeV}$ through mixing with a small Higgs coupling, but requires a significant degree of well-tempering. It is also possible to achieve $\Omega_{\chi}=\Omega_{\mathrm{DM}}$ with large Higgs couplings, particularly with $M_{D} \gtrsim 1 \mathrm{TeV}$, but avoiding direct detection constraints in such cases requires blind spot cancellations. While most of the parameter space that avoids the LUX bound requires little tuning, $\xi_{\mathrm{WT}}, \xi_{\mathrm{BS}}>0.1$, after XENON1T nearly all models either exhibit a significant degree of blind spot tuning, $\xi_{\mathrm{BS}}<0.1$, or must have mixing angles which are sensitively well-tempered to produce $\Omega_{\chi}=\Omega_{\mathrm{DM}}$ through coannihilation with small Higgs couplings with $\xi_{\mathrm{WT}}<0.1$. Thus, XENON1T strongly constrains the parameter space of singlet-doublet DM.

The constraints placed by direct detection on singlet-doublet scalar models are also substantial. In such models $\Omega_{\chi}=\Omega_{\mathrm{DM}}$ can be achieved at any mass through mixing for $M_{\chi}<$ $M_{D} \lesssim 550 \mathrm{GeV}$ with small DM-Higgs couplings, requiring significant well-tempering as in the fermionic case. For $M_{\chi} \gtrsim 550 \mathrm{GeV}$, however, annihilation through Higgs-mediated processes is required to accommodate $\Omega_{\chi}=\Omega_{\mathrm{DM}}$. Hence, these models require larger Higgs couplings and are subject to stronger direct detection bounds. Current limits from LUX place strong bounds up to large $M_{\chi}$ and XENON1T constrains almost the entire parameter space studied. In the examined parameter space, the few allowed regions needed significant coannihilation, with fine-tuning of $\xi_{\mathrm{BS}}<0.1$ to remain viable. However, a more comprehensive study of the full seven-dimensional parameter space might yield regions of lesser fine-tuning consistent with XENON1T.

The behavior of singlet-triplet scalar DM is qualitatively similar to that of singletdoublet DM. However, both gauge boson and Higgs mediated annihilation processes are quantitatively stronger. The correct relic density can be achieved through mixing alone for $M_{\chi}<$ $M_{T} \lesssim 1.9 \mathrm{TeV}$ though such processes, though the well-mixed region still requires significant well-tempering. Moreover, both the trilinear $h \chi \chi$ and quartic $h h \chi \chi$ interactions are stronger than in the singlet-doublet scalar case, enhancing annihilation even for smaller mixing angles and thus weakening direct detection bounds. LUX has little constraining power on the singlettriplet parameter space. XENON1T constraints are strong for vanishing or negative quartic couplings, allowing for minimal well-tempering only for nearly pure triplet models with $1.9 \mathrm{TeV} \lesssim M_{\chi} \lesssim 2.1 \mathrm{TeV}$. For positive quartic couplings, however, a significant region where $\mathrm{DM}$ is dominantly singlet and has minimal tuning of any sort, $\xi_{\mathrm{WT}}, \xi_{\mathrm{BS}}>0.1$, remains viable.

The present work has focused exclusively on experimental constraints from spin independent direct detection. However, many complementary probes exist. For example, even at cancellation points with vanishing DM-Higgs coupling, there will generically be $Z$-mediated spin dependent DM-nucleon scattering. Future direct detection probes [72-74] and both current and future constraints from neutrino telescopes [75-77] will place significant constraints on many of these models. Moreover, these models will also be constrained by astrophysical probes such as FERMI [78] and HESS [79]. We leave these analyses for future work. 


\section{Acknowledgments}

We are grateful to the Kavli Institute for Theoretical Physics at Santa Barbara and the Aspen Center for Physics, where part of this work was conducted. DS is supported in part by U.S. Department of Energy grant DE-FG02-92ER40701 and by the Gordon and Betty Moore Foundation through Grant No. 776 to the Caltech Moore Center for Theoretical Cosmology and Physics. CC is supported by a DOE Early Career Award DE-SC0010255.

\section{References}

[1] XENON100 collaboration, E. Aprile et al., Dark Matter Results from 100 Live Days of XENON100 Data, Phys. Rev. Lett. 107 (2011) 131302 [arXiv:1104.2549] [InSPIRE].

[2] XENON100 collaboration, E. Aprile et al., Dark Matter Results from 225 Live Days of XENON100 Data, Phys. Rev. Lett. 109 (2012) 181301 [arXiv:1207.5988] [INSPIRE].

[3] LUX collaboration, D. Akerib et al., First results from the LUX dark matter experiment at the Sanford Underground Research Facility, arXiv:1310.8214 [INSPIRE].

[4] XENON1T collaboration, E. Aprile, The XENON1T Dark Matter Search Experiment, arXiv: 1206.6288 [INSPIRE].

[5] D. Malling et al., After LUX: The LZ Program, arXiv:1110.0103 [INSPIRE].

[6] ATLAS collaboration, Observation of a new particle in the search for the Standard Model Higgs boson with the ATLAS detector at the LHC, Phys. Lett. B 716 (2012) 1 [arXiv: 1207.7214] [INSPIRE].

[7] CMS collaboration, Observation of a new boson at a mass of $125 \mathrm{GeV}$ with the CMS experiment at the LHC, Phys. Lett. B 716 (2012) 30 [arXiv:1207.7235] [INSPIRE].

[8] N. Arkani-Hamed, D.P. Finkbeiner, T.R. Slatyer and N. Weiner, A Theory of Dark Matter, Phys. Rev. D 79 (2009) 015014 [arXiv:0810.0713] [InSPIRE].

[9] M. Baumgart, C. Cheung, J.T. Ruderman, L.-T. Wang and I. Yavin, Non-Abelian Dark Sectors and Their Collider Signatures, JHEP 04 (2009) 014 [arXiv:0901.0283] [INSPIRE].

[10] C. Cheung, J.T. Ruderman, L.-T. Wang and I. Yavin, Kinetic Mixing as the Origin of Light Dark Scales, Phys. Rev. D 80 (2009) 035008 [arXiv:0902.3246] [INSPIRE].

[11] F. Chen, J.M. Cline and A.R. Frey, Nonabelian dark matter: models and constraints, Phys. Rev. D 80 (2009) 083516 [arXiv:0907.4746] [INSPIRE].

[12] A. Katz and R. Sundrum, Breaking the Dark Force, JHEP 06 (2009) 003 [arXiv:0902.3271] [INSPIRE].

[13] S. Andreas, M. Goodsell and A. Ringwald, Dark Matter and Dark Forces from a Supersymmetric Hidden Sector, Phys. Rev. D 87 (2013) 025007 [arXiv:1109.2869] [InSPIRE].

[14] D. Feldman, Z. Liu and P. Nath, The Stueckelberg Z-prime Extension with Kinetic Mixing and Milli-Charged Dark Matter From the Hidden Sector, Phys. Rev. D 75 (2007) 115001 [hep-ph/0702123] [INSPIRE].

[15] T. Cohen, D.J. Phalen, A. Pierce and K.M. Zurek, Asymmetric Dark Matter from a GeV Hidden Sector, Phys. Rev. D 82 (2010) 056001 [arXiv:1005.1655] [InSPIRE].

[16] D.P. Finkbeiner, T.R. Slatyer and N. Weiner, Nuclear scattering of dark matter coupled to a new light scalar, Phys. Rev. D 78 (2008) 116006 [arXiv:0810.0722] [INSPIRE].

[17] Y. Nomura and J. Thaler, Dark Matter through the Axion Portal, Phys. Rev. D 79 (2009) 075008 [arXiv: 0810 . 5397] [INSPIRE]. 
[18] Muon G-2 collaboration, G. Bennett et al., Final Report of the Muon E821 Anomalous Magnetic Moment Measurement at BNL, Phys. Rev. D 73 (2006) 072003 [hep-ex/0602035] [inSPIRE].

[19] M. Davier, A. Hoecker, B. Malaescu and Z. Zhang, Reevaluation of the Hadronic Contributions to the Muon g-2 and to alpha(MZ), Eur. Phys. J. C 71 (2011) 1515 [Erratum ibid. C 72 (2012) 1874] [arXiv: 1010.4180] [INSPIRE].

[20] F. Jegerlehner and R. Szafron, $\rho^{0}-\gamma$ mixing in the neutral channel pion form factor $F_{\pi}^{e}$ and its role in comparing $e^{+} e^{-}$with $\tau$ spectral functions, Eur. Phys. J. C 71 (2011) 1632 [arXiv:1101.2872] [INSPIRE].

[21] M. Cirelli, N. Fornengo and A. Strumia, Minimal dark matter, Nucl. Phys. B 753 (2006) 178 [hep-ph/0512090] [INSPIRE].

[22] M. Cirelli, A. Strumia and M. Tamburini, Cosmology and Astrophysics of Minimal Dark Matter, Nucl. Phys. B 787 (2007) 152 [arXiv:0706.4071] [INSPIRE].

[23] A. DiFranzo, K.I. Nagao, A. Rajaraman and T.M.P. Tait, Simplified Models for Dark Matter Interacting with Quarks, JHEP 11 (2013) 014 [arXiv:1308.2679] [INSPIRE].

[24] S. Chang, R. Edezhath, J. Hutchinson and M. Luty, Effective WIMPs, Phys. Rev. D 89 (2014) 015011 [arXiv: 1307.8120] [INSPIRE].

[25] Y. Bai and J. Berger, Fermion Portal Dark Matter, JHEP 11 (2013) 171 [arXiv:1308.0612] [INSPIRE].

[26] J. Goodman et al., Constraints on Light Majorana dark Matter from Colliders, Phys. Lett. B 695 (2011) 185 [arXiv: 1005.1286] [InSPIRE].

[27] P.J. Fox, R. Harnik, J. Kopp and Y. Tsai, Missing Energy Signatures of Dark Matter at the LHC, Phys. Rev. D 85 (2012) 056011 [arXiv:1109.4398] [INSPIRE].

[28] P.J. Fox, R. Harnik, J. Kopp and Y. Tsai, LEP Shines Light on Dark Matter, Phys. Rev. D 84 (2011) 014028 [arXiv: 1103.0240] [INSPIRE].

[29] R. Essig, J. Mardon, M. Papucci, T. Volansky and Y.-M. Zhong, Constraining Light Dark Matter with Low-Energy $e^{+} e^{-}$Colliders, JHEP 11 (2013) 167 [arXiv:1309.5084] [INSPIRE].

[30] M.R. Buckley, Asymmetric Dark Matter and Effective Operators, Phys. Rev. D 84 (2011) 043510 [arXiv: 1104 . 1429] [INSPIRE].

[31] Y.J. Chae and M. Perelstein, Dark Matter Search at a Linear Collider: Effective Operator Approach, JHEP 05 (2013) 138 [arXiv:1211.4008] [INSPIRE].

[32] I.M. Shoemaker and L. Vecchi, Unitarity and Monojet Bounds on Models for DAMA, CoGeNT and CRESST-II, Phys. Rev. D 86 (2012) 015023 [arXiv:1112.5457] [InSPIRE].

[33] G. Busoni, A. De Simone, E. Morgante and A. Riotto, On the Validity of the Effective Field Theory for Dark Matter Searches at the LHC, Phys. Lett. B 728 (2014) 412 [arXiv: 1307.2253] [INSPIRE].

[34] O. Buchmueller, M.J. Dolan and C. McCabe, Beyond Effective Field Theory for Dark Matter Searches at the LHC, JHEP 01 (2014) 025 [arXiv: 1308.6799] [INSPIRE].

[35] N. Arkani-Hamed, A. Delgado and G. Giudice, The well-tempered neutralino, Nucl. Phys. B 741 (2006) 108 [hep-ph/0601041] [INSPIRE].

[36] H. Baer, A. Mustafayev, E.-K. Park and X. Tata, Target dark matter detection rates in models with a well-tempered neutralino, JCAP 01 (2007) 017 [hep-ph/0611387] [INSPIRE].

[37] J.L. Feng and D. Sanford, Heart of Darkness: The Significance of the Zeptobarn Scale for Neutralino Direct Detection, JCAP 05 (2011) 018 [arXiv:1009.3934] [INSPIRE].

[38] J.L. Feng, K.T. Matchev and F. Wilczek, Neutralino dark matter in focus point supersymmetry, Phys. Lett. B 482 (2000) 388 [hep-ph/0004043] [INSPIRE]. 
[39] H. Baer, T. Krupovnickas, S. Profumo and P. Ullio, Model independent approach to focus point supersymmetry: From dark matter to collider searches, JHEP 10 (2005) 020 [hep-ph/0507282] [INSPIRE].

[40] J.L. Feng, K.T. Matchev and D. Sanford, Focus Point Supersymmetry Redux, Phys. Rev. D 85 (2012) 075007 [arXiv: 1112.3021] [INSPIRE].

[41] P. Draper, J. Feng, P. Kant, S. Profumo and D. Sanford, Dark Matter Detection in Focus Point Supersymmetry, Phys. Rev. D 88 (2013) 015025 [arXiv:1304.1159] [INSPIRE].

[42] A. Biswas, D. Majumdar, A. Sil and P. Bhattacharjee, Two Component Dark Matter: A Possible Explanation of $130 \mathrm{GeV} \gamma-$ Ray Line from the Galactic Centre, JCAP 12 (2013) 049 [arXiv: 1301.3668] [INSPIRE].

[43] S. Bhattacharya, A. Drozd, B. Grzadkowski and J. Wudka, Two-Component Dark Matter, JHEP 10 (2013) 158 [arXiv:1309.2986] [INSPIRE].

[44] G. Bélanger, F. Boudjema, A. Pukhov and A. Semenov, MicrOMEGAs 2.0: A Program to calculate the relic density of dark matter in a generic model, Comput. Phys. Commun. 176 (2007) 367 [hep-ph/0607059] [INSPIRE].

[45] G. Bélanger, F. Boudjema, A. Pukhov and A. Semenov, Dark matter direct detection rate in a generic model with MicrOMEGAs 2.2, Comput. Phys. Commun. 180 (2009) 747 [arXiv:0803.2360] [INSPIRE].

[46] G. Bélanger et al., Indirect search for dark matter with MicrOMEGAs2.4, Comput. Phys. Commun. 182 (2011) 842 [arXiv: 1004.1092] [INSPIRE].

[47] N.D. Christensen and C. Duhr, FeynRules - Feynman rules made easy, Comput. Phys. Commun. 180 (2009) 1614 [arXiv:0806.4194] [InSPIRE].

[48] Planck collaboration, P. Ade et al., Planck 2013 results. XVI. Cosmological parameters, arXiv: 1303.5076 [INSPIRE].

[49] C. Cheung, L.J. Hall, D. Pinner and J.T. Ruderman, Prospects and blind spots for neutralino dark matter, JHEP 05 (2013) 100 [arXiv:1211.4873] [INSPIRE].

[50] R.J. Hill and M.P. Solon, Standard Model anatomy of WIMP dark matter direct detection I: weak-scale matching, arXiv:1401.3339 [INSPIRE].

[51] R.J. Hill and M.P. Solon, WIMP-nucleon scattering with heavy WIMP effective theory, arXiv:1309.4092 [INSPIRE].

[52] J. Giedt, A.W. Thomas and R.D. Young, Dark matter, the CMSSM and lattice QCD, Phys. Rev. Lett. 103 (2009) 201802 [arXiv:0907.4177] [INSPIRE].

[53] T. Cohen, J. Kearney, A. Pierce and D. Tucker-Smith, Singlet-Doublet Dark Matter, Phys. Rev. D 85 (2012) 075003 [arXiv:1109.2604] [InSPIRE].

[54] A. Joglekar, P. Schwaller and C.E. Wagner, Dark Matter and Enhanced Higgs to Di-photon Rate from Vector-like Leptons, JHEP 12 (2012) 064 [arXiv:1207.4235] [INSPIRE].

[55] V. Silveira and A. Zee, Scalar phantoms, Phys. Lett. B 161 (1985) 136 [inSPIRE].

[56] J. McDonald, Gauge singlet scalars as cold dark matter, Phys. Rev. D 50 (1994) 3637 [hep-ph/0702143] [INSPIRE].

[57] C. Burgess, M. Pospelov and T. ter Veldhuis, The Minimal model of nonbaryonic dark matter: A Singlet scalar, Nucl. Phys. B 619 (2001) 709 [hep-ph/0011335] [InSPIRE].

[58] C. Boehm and P. Fayet, Scalar dark matter candidates, Nucl. Phys. B 683 (2004) 219 [hep-ph/0305261] [INSPIRE].

[59] A. Biswas and D. Majumdar, The Real Gauge Singlet Scalar Extension of Standard Model: A Possible Candidate of Cold Dark Matter, Pramana 80 (2013) 539 [arXiv:1102.3024] [INSPIRE]. 
[60] A. Drozd, B. Grzadkowski and J. Wudka, Multi-Scalar-Singlet Extension of the Standard Model - the Case for Dark Matter and an Invisible Higgs Boson, JHEP 04 (2012) 006 [arXiv:1112.2582] [INSPIRE].

[61] J.M. Cline, K. Kainulainen, P. Scott and C. Weniger, Update on scalar singlet dark matter, Phys. Rev. D 88 (2013) 055025 [arXiv:1306.4710] [InSPIRE].

[62] E. Ma, Verifiable radiative seesaw mechanism of neutrino mass and dark matter, Phys. Rev. D 73 (2006) 077301 [hep-ph/0601225] [INSPIRE].

[63] L. Lopez Honorez, E. Nezri, J.F. Oliver and M.H. Tytgat, The inert doublet model: an archetype for dark matter, JCAP 02 (2007) 028 [hep-ph/0612275] [INSPIRE].

[64] M. Kadastik, K. Kannike and M. Raidal, Matter parity as the origin of scalar Dark Matter, Phys. Rev. D 81 (2010) 015002 [arXiv:0903.2475] [INSPIRE].

[65] M. Kadastik, K. Kannike and M. Raidal, Dark Matter as the signal of Grand Unification, Phys. Rev. D 80 (2009) 085020 [Erratum ibid. D 81 (2010) 029903] [arXiv:0907.1894] [INSPIRE].

[66] P. Fileviez Perez, H.H. Patel, M. Ramsey-Musolf and K. Wang, Triplet Scalars and Dark Matter at the LHC, Phys. Rev. D 79 (2009) 055024 [arXiv:0811.3957] [INSPIRE].

[67] O. Fischer and J. van der Bij, Multi-singlet and singlet-triplet scalar dark matter, Mod. Phys. Lett. A 26 (2011) 2039 [INSPIRE].

[68] O. Fischer and J.J. van der Bij, The scalar Singlet-Triplet Dark Matter Model, arXiv: 1311.1077 [INSPIRE].

[69] U. Chattopadhyay, D. Choudhury, M. Drees, P. Konar and D. Roy, Looking for a heavy Higgsino LSP in collider and dark matter experiments, Phys. Lett. B 632 (2006) 114 [hep-ph/0508098] [INSPIRE].

[70] J. Espinosa, C. Grojean, M. Muhlleitner and M. Trott, First Glimpses at Higgs' face, JHEP 12 (2012) 045 [arXiv: 1207.1717] [INSPIRE].

[71] T. Hambye, F.-S. Ling, L. Lopez Honorez and J. Rocher, Scalar Multiplet Dark Matter, JHEP 07 (2009) 090 [Erratum ibid. 1005 (2010) 066] [arXiv: 0903.4010] [INSPIRE].

[72] COUPP collaboration, E. Ramberg, Developing a 60-kg bubble chamber for dark matter detection, Nucl. Instrum. Meth. A 623 (2010) 454 [INSPIRE].

[73] XMASS collaboration, J. Liu, Construction and performance of XMASS 800kg detector, Nucl. Phys. Proc. Suppl. 229-232 (2012) 564 [INSPIRE].

[74] CDMS collaboration, T. Bruch, CDMS-II to SuperCDMS: WIMP search at a zeptobarn, arXiv: 1001.3037 [INSPIRE].

[75] ICECUBE collaboration, M. Aartsen et al., Search for dark matter annihilations in the Sun with the 79-string IceCube detector, Phys. Rev. Lett. 110 (2013) 131302 [arXiv:1212.4097] [INSPIRE].

[76] ICECUBE collaboration, R. Abbasi et al., Multi-year search for dark matter annihilations in the Sun with the AMANDA-II and IceCube detectors, Phys. Rev. D 85 (2012) 042002 [arXiv:1112.1840] [INSPIRE].

[77] IcECuBE collaboration, R. Abbasi et al., Search for Dark Matter from the Galactic Halo with the IceCube Neutrino Observatory, Phys. Rev. D 84 (2011) 022004 [arXiv:1101.3349] [INSPIRE].

[78] Fermi-LAT collaboration, M. Ackermann et al., Constraining Dark Matter Models from a Combined Analysis of Milky Way Satellites with the Fermi Large Area Telescope, Phys. Rev. Lett. 107 (2011) 241302 [arXiv:1108.3546] [INSPIRE].

[79] H.E.S.S. collaboration, A. Abramowski et al., Search for photon line-like signatures from Dark Matter annihilations with H.E.S.S, Phys. Rev. Lett. 110 (2013) 041301 [arXiv:1301.1173] [INSPIRE]. 